PPPL-1938 (Nov. 1982)

Physica 8D(3), 360-380 (Sept. 1983)

\title{
LONG-TIME CORRELATIONS IN THE STOCHASTIC REGIME
}

\author{
Charles F. F. KARNEY \\ Plasma Physics Laboratory, Princeton University, \\ Princeton, New Jersey 08544, USA
}

The phase space for Hamiltonians of two degrees of freedom is usually divided into stochastic and integrable components. Even when well into the stochastic regime, integrable orbits may surround small stable regions or islands. The effect of these islands on the correlation function for the stochastic trajectories is examined. Depending on the value of the parameter describing the rotation number for the elliptic fixed point at the center of the island, the long-time correlation function may decay as $t^{-5}$ or exponentially, but more commonly it decays much more slowly (roughly as $t^{-1}$ ). As a consequence these small islands may have a profound effect on the properties of the stochastic orbits. In particular, there is evidence that the evolution of a distribution of particles is no longer governed by a diffusion equation.

Key words: long-time correlations, area-preserving mappings, diffusion, numerical experiments.

This paper was reprinted in Hamiltonian Dynamical Systems, edited by R. S. MacKay and J. D. Meiss (Adam-Hilger, Bristol, 1987), pages 585-605. 


\section{Introduction}

Many important problems in physics are described by Hamiltonians of two degrees of freedom. Examples are the motion of a charged particle in electrostatic waves, the motion of a charged particle in various magnetic confinement devices, the acceleration of a particle bouncing between a fixed and an oscillating wall, the wandering of magnetic field lines, etc. In such systems, there may be a parameter $k$ which governs the strength of the coupling between the two degrees of freedom. When $k$ is zero (no coupling), the system is integrable. As $k$ is increased, some of the integrable trajectories disappear, and the motion becomes stochastic. Eventually, when the $k$ is large, nearly all of phase space is occupied by stochastic trajectories. These properties are nicely illustrated by the standard mapping [1]. Although this mapping is an idealization, it accurately portrays many of the properties of real systems.

When $k$ is small, various perturbation theories are available to describe the trajectories approximately. On the other hand, in the highly stochastic regime ( $k$ large) certain statistical quantities may be analytically determined by assuming that the motion is ergodic and

that the correlation time is short. In the case of the standard mapping, this allows simple determinations of quantities such as the diffusion coefficient [1,2] and the KS-entropy [1].

The purpose of this paper is to examine more critically the assumption that the correlation time is short in the stochastic regime. Our interest in this problem was triggered by studies of the diffusion coefficient for the standard mapping,

$$
r_{t}-r_{t-1}=-k \sin \theta_{t-1}, \quad \theta_{t}-\theta_{t-1}=r_{t} .
$$

Far above the stochasticity threshold $k \gg 1$, the diffusion coefficient defined as

$$
\mathcal{D}=\lim _{t \rightarrow \infty} \frac{\left\langle\left(r_{t}-r_{0}\right)^{2}\right\rangle}{2 t},
$$

where the average is over some appropriate ensemble, is given by assuming that $\theta$ is a random variable in the equation for $r$. This is equivalent to assuming that the correlation function is proportional to a delta function and gives the "quasi-linear" result $\mathcal{D} / \mathcal{D}_{\mathrm{ql}}=\frac{1}{4} k^{2}$. Including the correlations out to short times (about $t=4$ ) gives corrections to the diffusion coefficient reported by Rechester and White [2] which can enhance the diffusion coefficient by as much as a factor of about two. However, a numerical determination [3] of the diffusion at $k=6.6$ gave $\mathcal{D} / \mathcal{D}_{\mathrm{ql}} \sim 80$. At this value of $k$ there is an island ("accelerator mode") present in the stochastic sea. Although the orbits used to compute $\mathcal{D}$ were all in the stochastic region, they were able to wander close to the island and stay close to it for a long time. This introduces long-time correlations into the motion and accounts for the anomalously large value of $\mathcal{D}$ observed.

The appearance of islands in the stochastic regime is not at all unusual. This may be seen from Sinai's estimate [1] for the number $\nu(T)$ of periodic orbits of period $\leq T$ : $\nu(T) \sim \exp (h T)$ for $T \rightarrow \infty$ where $h$ is the KS-entropy. Now the majority of these periodic orbits are unstable. However as $k$ is increased, $h$ generally increases $\left(h \approx \log \left(\frac{1}{2} k\right)\right.$ [1] for the standard mapping) and hence new periodic orbits must appear. Generally a tangent bifurcation is responsible for the appearance of the new periodic orbits. At the tangent bifurcation, a pair of stable and unstable periodic orbits is born. The stable orbit gives rise to other longer periodic orbits as $k$ is increased and eventually becomes unstable. Thus as we increase $k$, many small islands appear via tangent bifurcations, survive for some interval of $k$, and finally disappear (usually through period doubling). If we pick a particular value of $k$, it is not clear that there will necessarily be any islands present. However, we may speculate that at some arbitrarily close value of $k$, there will be some islands. 
It may be objected that the large effect seen in the standard mapping arises because the islands are accelerator modes [1] and that such islands are a rather special feature of the standard mapping. While accelerator modes are the only islands which will contribute significantly to the force (i.e., acceleration) correlation function and hence to the diffusion coefficient, any islands will contribute to the correlation of some functions on phase space. The results of this study will be applicable to systems like the Fermi map which have no accelerator modes. This study also contributes to the understanding of the more general problem of motion in a divided phase space.

In this paper, we wish to examine more closely the effect these islands have on a stochastic trajectory. As far as determining the effect on the correlation function, this involves determining how "sticky" the island is. Given that the stochastic trajectory comes within a certain distance of the boundary of the island, how long do we expect it to stay close to the island? This approach is inspired by work of Channon and Lebowitz [4] on the correlations of a trajectory in the stochastic band trapped between two KAM surfaces in the Hénon map. Similar work has been carried out on the whisker map by Chirikov and Shepelyansky [5]. This work is being extended by B. V. Chirikov and F. Vivaldi.

Since we concentrate only on the behavior close to the island, this approach may be characterized as a local one. This should be compared with Fourier transform methods [2], which are global and are not well suited to the description of localized phenomena. For instance, Meiss et al. [6] attempted to use such methods to compute the long-time correlations for the standard mapping, and they found poor agreement with numerical experiments whenever islands were present.

The paper is organized as follows. In section 2, we derive a canonical mapping which describes the behavior near an island. Next (section 3), we define the trapping statistics which describe how sticky the island is. The results for the trapping statistics are given in sections 4 and 5 . In section 6 , we show how to apply these results to obtaining the correlation function. The results are discussed in section 7 .

\section{Derivation of mapping.}

Far into the stochastic regime for a general mapping, the islands which appear via tangent bifurcations are very small and exist only for a small interval in parameter space. This allows us to approximate them by a Taylor expansion in both phase and parameter space about the tangent bifurcation point retaining only the leading terms. This was carried out in ref. 3 where the resulting mapping was reduced to a canonical form

$$
Q: \quad y_{t}-y_{t-1}=2\left(x_{t-1}^{2}-K\right) \equiv g\left(x_{t-1} ; K\right), \quad x_{t}-x_{t-1}=y_{t} .
$$

Here $K$ is proportional to $k-k_{\text {tang }}\left(k_{\text {tang }}\right.$ is the parameter value where the tangent bifurcation take place) and $x$ and $y$ are related to the original phase space coordinates by a smooth transformation. The mapping $Q$ represents an approximation of the general mapping close to the point of tangent bifurcation. For $K<1, Q$ has no periodic orbits. At $K=0$, there is a tangent bifurcation when an unstable fixed point appears at $x=y=0$. (This is not a hyperbolic point since its stability is determined by the quadratic terms in the mapping.) For $0<K<1$, this fixed point splits into a pair of stable (elliptic) and unstable (hyperbolic) fixed points located at $(x, y)=(\mp \sqrt{K}, 0)$, respectively. The elliptic fixed point is usually surrounded by integrable trajectories (KAM curves) which define a stable region (the island) in which the motion is bounded. An example of island structure is shown in fig. 
1 for $K=0.1$ (the value of $K$ at which extensive numerical calculations have carried out). At $K=1$ the stable fixed point becomes unstable and gives rise to a period-2 orbit via a period-doubling bifurcation. At $K=1.2840$ the period-doubling sequence accumulates [7], and at this point (or shortly hereafter [8]) the area of the stable regions becomes very small. For $0<K<1$, the mapping $Q$ may be transformed to the Hénon quadratic map [9], the parameter $K$ being related to Hénon's $\cos \alpha$ by

$$
\cos \alpha=1-2 \sqrt{K}
$$

( $\alpha$ is the mean angle of rotation for points close to the stable fixed point).

Referring again to the islands shown in fig. 1 , consider a particle which at $t=0$ is close to, but outside, the islands. (We often speak of an orbit in terms of the position of a particle whose equation of motion is given by $Q$.) Initially, the particle will stay close to the islands; however as we let $t \rightarrow \pm \infty$, we find $(x, y) \rightarrow(\infty, \pm \infty)$. It is just such trajectories we are interested in, because they correspond to particles in the stochastic region of the general mapping approaching the islands, staying there for some time (and contributing to long-time correlations), and then escaping back to the main part of the stochastic region.

What we want to do is to follow such trajectories numerically and see how long they stay close to the islands. However, we need some method of fairly sampling these trajectories. "Fairly" means that we should sample them in the same way that a stochastic trajectory of a general mapping does. Since the stochastic trajectory is ergodic over the connected stochastic region of phase space, we must sample trajectories in the same way; i.e., we must ensure that the superposition of all the sampled trajectories covers the region outside the islands uniformly.

We achieve this by changing $Q$ so that the phase space is compact. This may be accomplished by replacing $g(x ; K)$ in $Q$ by the periodic function

$$
g^{*}(x ; K) \equiv \begin{cases}g(x ; K) & \text { for } x_{\min } \leq x<x_{\max }, \\ g(x \pm L ; K) & \text { otherwise, }\end{cases}
$$

where $L=x_{\max }-x_{\min }>0$. The resulting map will be called $Q^{*}$. If $x_{\min }$ and $x_{\max }$ are chosen to span the region where there are islands in $Q$, then $Q^{*}$ obviously will contain the same islands. Furthermore the motion close to the islands will be the same. By replacing $g$ by $g^{*}$, the whole of phase space becomes periodic in the $x$ and $y$ directions with period $L$. The motion can be treated as though it were on a torus. A particle which starts near the island will, as with $Q$, spend some time close to the island. But when it moves away from the island it no longer goes to infinity, but rather it loops around the torus and has another chance to approach the islands. Since $Q^{*}$ is area-preserving, this single orbit will ergodically cover the region outside the islands in the desired manner. Examples of such orbits are shown in fig. 2 for the same parameters as for fig. 1. (One embarassing feature of $Q^{*}$ is that new islands are introduced. They are in fact accelerator modes - a particle inside one of them loops around the torus in either the $x$ or $y$ directions. As discussed in appendix A, these islands do not effect the statistics for the long trapping times.)

One useful way of looking at $Q^{*}$ is as a magnification of a small region near a tangent bifurcation in the general mapping. The difference is that once the trajectory leaves the vicinity of the islands, it is immediately re-injected on the other side of the islands. In the general map, the trajectory will spend some long time, which depends on the ratio of the size of the islands to the total accessible portion of phase space, in the stochastic sea before coming back to the vicinity of the islands. 
Assuming that the long-time behavior of stochastic orbits is dominated by the region close to the islands, there are two advantages to reducing the problem to a study of $Q^{*}$. Firstly, since $Q^{*}$ describes the behavior of most islands far into the stochastic regime, the properties of many mappings may be treated by looking at a special mapping $Q^{*}$ which depends only on a single parameter $K$. The second advantage is that the properties of orbits close to the islands may be studied much more efficiently because there is no need to follow orbits while they spend a long and uninteresting time far from the islands.

\section{The trapping statistics.}

The prescription for numerically determining how sticky is the island system in $Q$ for a given $K$ is to pick trajectories outside the islands in $Q^{*}$ and to iterate the mapping many times. The mapping is performed on the torus, i.e., $x$ and $y$ are reduced to their base intervals $\left[x_{\min }, x_{\max }\right)$ and $\left[-\frac{1}{2} L, \frac{1}{2} L\right)$ after each iteration. However, we keep track of when an orbit moves off one edge and re-appears at the opposite edge. The orbit is then divided at those points when the orbit looped around the torus, and the lengths of the resulting orbit segments are recorded. The main result of such a calculation are then the trapping statistics $f_{t}$ which are proportional to the number of orbit segments which have a length of $t$.

Suppose that the total length of the orbit is $T$ and $N_{t}$ is the number of segments of length $t$. If $T$ is so large that we can ignore partial segments at the ends of the orbit (this problem is examined below), then we have $\sum t N_{t}=T$; the total number of segments is $N=\sum N_{t}$. The trapping statistics are defined by $f_{t}=N_{t} / T$ and are therefore normalized so that $\sum t f_{t}=1$. The mean length of the orbits is given by $\alpha=1 / \sum f_{t}(=T / N)$. The probability that a particular segment has length $t$ is $p_{t}=\alpha f_{t}\left(=N_{t} / N\right)$. If an arbitrary point is chosen in the orbit, then $t f_{t}$ is the probability that this point belongs to a segment of length $t$ and $f_{t}$ is the probability that it belongs to the beginning, say, of a segment of length $t$.

Three factors effect the measurement of $f_{t}$. They are (a) the presence of the spurious accelerator modes, (b) the choice of $x_{\min }$ and $x_{\max }$, and (c) the total length $T$ of the trajectory used to measure $f_{t}$. The first two items only effect $f_{t}$ for small $t$ (apart from an overall normalization). In order to account for the last item, we define $f_{t}$ by $N_{t} /(T+1-t)$ (rather than $N_{t} / T$ ). This accounts for the fact that we are less likely to observe orbit segments whose length is close to $T$. All these points are discussed in detail in appendix A.

The survival probability

$$
P_{t}=\sum_{\tau=t+1}^{\infty} p_{\tau}
$$

is the probability that an orbit beginning in a segment at $t=0$ is still trapped in the same segment at time $t$. Note that $P_{0}=1$ as required. This is the quantity studied in refs. 4 and 5 . The correlation function

$$
C_{\tau}=\sum_{t=\tau}^{\infty}(t-\tau) f_{t}=\sum_{t=\tau}^{\infty} P_{t} / \alpha
$$

is the probability that a particle is trapped in the same segment at two times $\tau$ apart. Again, we have $C_{0}=1$. 
There are two other ways of interpreting $C_{\tau}$. If we start many particles at positions uniformly distributed in the stochastic sea of $Q^{*}$ (i.e., in the dark region of fig. 2), then $C_{\tau}$ gives the fraction of particles remaining in the $L \times L$ square after $\tau$ iterations of $Q$ (rather than $Q^{*}$ ). Alternatively, consider a drunkard who executes a one-dimensional random walk with velocity $v=d r / d t= \pm 1$. The direction of each step is chosen randomly, while the durations of the steps are chosen to be the lengths of consecutive trapped segments of $Q^{*}$. Then for integer $\tau, C_{\tau}$ is just the usual correlation function for such a process, i.e., $\left\langle v_{t} v_{t+\tau}\right\rangle_{t}$. The behavior of this random-walk process is similar to the behavior of an orbit in the general mapping when two accelerator modes with opposite values of the acceleration are present. (This is the case with the first-order accelerator modes for the standard mapping.) In section 6 , we will show how $C_{\tau}$ is related to the correlation function for the general mapping.

A diffusion coefficient may be defined by

$$
D=\frac{1}{2} C_{0}+\sum_{\tau=1}^{\infty} C_{\tau}=\sum \frac{1}{2} t^{2} f_{t} .
$$

This gives the diffusion rate for the drunkard in the random-walk problem above. It is also related to the diffusion coefficient for the general mapping (see section 6).

Since $Q^{*}$ must be iterated many times to provide good statistics for $f_{t}$ for large $t$, extraordinary steps were taken to ensure that the numerical program was fast and reliable. The time for one iteration on a Cray- 1 is a little less than 75 ns. One way that the code was made more reliable was by doing the arithmetic in fixed-point (as opposed to floating-point) notation. The numerical mapping is then one-to-one which is the discrete counterpart of area-preserving. (Floating-point realizations of mappings are typically many-to-one.) This precludes the possibility of an orbit, which starts far from the island, approaching the island and becoming permanently trapped near the island. Even though such behavior is forbidden for an area-preserving mapping, such behavior may be observing with a floatingpoint realization. Details of the numerical methods are given in appendix B.

\section{The results for small $K$.}

We begin by considering the cases where $K$ is small or zero. In this case the mapping equations are nearly integrable and this enables us to derive approximate analytic expressions for $f_{t}$. Figure 3 shows $f_{t}$ for $K=-10^{-4}, 0$, and $10^{-4}$. Three types of behavior are

seen for $t \rightarrow \infty$ : a cutoff distribution $f_{t}=0$ for $t>t_{\max } \approx 50$, a rapid algebraic decay $f_{t} \sim t^{-7}$, and an exponential decay $f_{t} \sim \exp (-0.1 t)$.

The easiest case to begin with is $K=0$. The method for deriving $f_{t}$ analytically consists of computing the length of the trajectory through some point and then assigning some probability that this trajectory will be chosen. The first part of the calculation has been carried out by Zisook [10]. We repeat the calculation here to establish the method for other cases.

When $K=0$, we are exactly at the tangent bifurcation point. There are no islands in this case, but trajectories can still spend arbitrarily long near the fixed point at $(x, y)=$ $(0,0)$. Near this point $x_{t}-x_{t-1}$ and $y_{t}-y_{t-1}$ are small. We therefore rescale $x, y$, and $t$ with $x=\epsilon^{\alpha} X, y=\epsilon^{\beta} Y, t=\epsilon^{-1} T$ where $\epsilon$ is small. The mapping $Q$ becomes 


$$
\begin{aligned}
& \frac{Y(T)-Y(T-\epsilon)}{\epsilon}=\epsilon^{2 \beta-\alpha-1} 2 X^{2}(T-\epsilon), \\
& \frac{X(T)-X(T-\epsilon)}{\epsilon}=\epsilon^{\alpha-\beta-1} Y(T) .
\end{aligned}
$$

Choosing $\alpha=3$ and $\beta=2$ and replacing the left hand sides by derivatives, we obtain

$$
\frac{d Y}{d T}=2 X^{2}, \quad \frac{d X}{d T}=Y .
$$

These are Hamilton's equations (with $X$ and $Y$ being conjugate position and momentum coordinates) for the Hamiltonian

$$
H=\frac{1}{2} Y^{2}-\frac{2}{3} X^{3} .
$$

Curves of constant $H$ in the $(X, Y)$ plane give the trajectories, examples of which are shown in fig. 4(a). We define the trapping time as the time it takes to traverse one of these curves from $Y=-\infty$ to $\infty$. (This Hamiltonian gives escape to infinity in a finite time. The time taken for a particle to escape to infinity in $Q$ is infinite, but very weakly so. The particle reaches $y$ from $y=0$ in roughly $\log \log y$ steps for $y$ large.) Since $d X / d T=Y=$ $\sqrt{2\left(H+\frac{2}{3} X^{3}\right)}$, the trapping time may be written as

$$
T\left(X_{0}\right)=2 \int_{X_{0}}^{\infty} \frac{d X}{\sqrt{\frac{4}{3}\left(X^{3}-X_{0}^{3}\right)}},
$$

where $X_{0}=-\left(\frac{3}{2} H\right)^{1 / 3}$ is the $X$ intercept of the trajectory with $Y=0$. Performing the integration gives

$$
T\left(X_{0}\right)=\left\{\begin{array}{c}
1 \\
\sqrt{3}
\end{array}\right\} \times 4.207\left|X_{0}\right|^{-1 / 2}, \quad \text { for } X \gtrless 0 .
$$

(The numbers here may be written in terms of incomplete elliptic integrals.)

This completes the computation of the trapping time. We now assign weights to each trapping time by requiring that particles spend equal times in equal areas of phase space. Let $A\left(X_{0}\right)$ be the area between the trajectory passing through the origin and that passing through $\left(X_{0}, 0\right)$ in fig. 4(a). Since $Y \sim X^{3 / 2}$, scaling invariance gives $A\left(X_{0}\right)=A(1) X_{0}^{5 / 2}$. (This procedure needs to be carried out separately for positive and negative $X_{0}$. However, the scaling relations are the same in the two cases.) Parameterizing in terms of the trapping time $T$ gives $A(T) \sim T^{-5}$. The fraction of particles which are trapped for times between $T$ and $T+d T$ is proportional to the differential area $d A(T) \sim T^{-6} d T$. Finally, we divide by $T$ to give $T^{-7} d T$ as the number of orbit segments of lengths in this range. In unscaled variables, we have $f_{t} \sim t^{-7}$ which is valid for $t$ large. The correlation function has the asymptotic form $C_{\tau} \sim \tau^{-5}$.

It is interesting to enquire what the asymptotic behavior for $f_{t}$ would be if $g$ in (2) were a higher-order polynomial in $x$. If $g(x ; 0)=x^{m}$, with $m>1$, we can repeat the above calculation and find that

$$
f_{t} \sim \frac{1}{t^{(3 m+1) /(m-1)}}
$$

Since $f_{t}$ has a finite second moment, $D$ always exists. (For $m=1$, we find an exponential decay of $f_{t}$. This corresponds to the case $K>0$ discussed below.) 
When $K$ is small and negative, there are no periodic orbits. The particle can spend only a bounded time close to the origin. Defining scaled variables as before, together with $K=-\epsilon^{4}$, gives differential equations which are derivable from the Hamiltonian

$$
H=\frac{1}{2} Y^{2}-\frac{2}{3} X^{3}-2 X .
$$

The trapping time is now

$$
T\left(X_{0}\right)=\int_{X_{0}}^{\infty} \frac{d X}{\sqrt{\frac{1}{3} X^{3}+X-\frac{1}{3} X_{0}^{3}-X_{0}}},
$$

where $X_{0}$ is the $X$ intercept of the trajectory with $Y=0$, i.e., it is the real root of $H+\frac{2}{3} X_{0}^{3}+$ $2 X_{0}=0$. This is plotted as a function of $X_{0}$ in fig. $5(\mathrm{a})$. We see it attains a maximum value of $T_{\max }=5.1454$ at $X_{0}=-0.5536$. In unscaled variables this means that the maximum trapping time is $t_{\max }=5.1454|K|^{-1 / 4}$ and that this trapping time is attained by particles passing through $(x, y)=(-0.5536 \sqrt{|K|}, 0)$. The $|K|^{-1 / 4}$ scaling of the maximum trapping time has been derived by Zisook [10].

To assign probabilities to the various trapping times, we define $A\left(X_{0}\right)$ as the area between the trajectory passing through $(0,0)$ and that passing through $\left(X_{0}, 0\right)$. Since $Y=$ $2 \sqrt{\frac{1}{3} X^{3}+X-\frac{1}{3} X_{0}^{3}-X_{0}}$, we obtain

$$
A\left(X_{0}\right)=4 \int_{0}^{\infty} \sqrt{\frac{1}{3} X^{3}+X} d X-4 \int_{X_{0}}^{\infty} \sqrt{\frac{1}{3} X^{3}+X-\frac{1}{3} X_{0}^{3}-X_{0}} d X .
$$

(The two integrals need to be done to together to get a finite answer.) Differentiating gives

$$
d A\left(X_{0}\right) / d X_{0}=2\left(X_{0}^{2}+1\right) T\left(X_{0}\right)
$$

The number of orbit segments of length $T$ is then proportional to

$$
F(T) \equiv \frac{1}{T} \frac{d A\left(X_{0}\right)}{d X_{0}} /\left|\frac{d T\left(X_{0}\right)}{d X_{0}}\right|=\sum \frac{2\left(X_{0}^{2}+1\right)}{\left|T^{\prime}\left(X_{0}\right)\right|} .
$$

The right hand side is written as a function of $X_{0}$. This is converted to a function of $T$ by inverting $T\left(X_{0}\right)$. Since this gives a double-valued function, the two branches must be summed over as indicated by the summation sign. In unscaled variables we have

$$
f_{t} \sim F\left(|K|^{1 / 4} t\right)
$$

for $t=O\left(|K|^{-1 / 4}\right)$. The function $F(T)$ is plotted in fig. $5(\mathrm{~b})$. For $T \ll T_{\max }$, we have $F(T) \approx 6.205 \times 10^{5} T^{-7}$ while for $T \approx T_{\max }, F(T) \approx 3.024\left(T_{\max }-T\right)^{-1 / 2}$. The correlation function is given by the second integration of $f_{t}$ so that for $\tau \approx t_{\max }$ we have $C_{\tau} \sim\left(t_{\max }-\right.$ $\tau)^{3 / 2}$ for $\tau \leq t_{\max }$.

Figure 5(b) should be compared with fig. 3(a). The effect of the singularity in $F(T)$ at $T_{\max }$ is evident, although its position isn't quite right. Furthermore, the decay of $f_{t}$ for smaller times is somewhat slower (approximately as $t^{-6}$ ) than for $F(T)$. These discrepancies arise because we are not far enough into the asymptotic regime since $\epsilon$ is not very small (0.1). The formula $t_{\max }=5.1454|K|^{-1 / 4}$ may easily be verified for smaller values of $|K|$. The $T^{-7}$ behavior of $F(T)$ has of course the same origin as that of $f_{t}$ for $K=0$. However the numerical results for $K=0$ given in fig. $3(\mathrm{~b})$ show that it is not attained until about $t \sim 100$. When $K=-10^{-4}, t_{\max }$ is only about 50 , and there is no interval in which the $t^{-7}$ behavior is exhibited. 
Finally we turn to the case $K>0$. As with $K<0$, we can approximately derive the motion from the Hamiltonian

$$
H=\frac{1}{2} Y^{2}-\frac{2}{3} X^{3}+2 X,
$$

where we have used the same scaled variables as previously except that $K=\epsilon^{4}$. The trajectories for this Hamiltonian are given in fig. 4(b). There is single island centered at the fixed point at $(X, Y)=(-1,0)$ and the island extends all the way to the separatrix emanating from the unstable fixed point at $(X, Y)=(1,0)$. This is an idealization because for the mapping there is a stochastic band close to the separatrix. However, for small $K$ this band is very thin, and the island does have quite a "clean" outer edge.

We may carry out the analysis given for $K<0$ with appropriate changes to obtain $f_{t}$ analytically. However, we may avoid doing a lot of tedious integrals by concentrating only on the long-time behavior of $f_{t}$. For shorter times, namely for $100 \lesssim t \ll K^{-1 / 4}$, we expect that $f_{t} \sim t^{-7}$ because the relevant trajectories never get close to the island and the Hamiltonian (11) approximately reduces to that for $K=0$ (7). Since only the region close to the unstable fixed point contributes to $f_{t}$ for large $t$, we need only consider this region. In addition, we should distinguish those trajectories which encircle the island and so encounter the region near the fixed point twice from those which do not and only encounter this region once. For a given distance from the fixed point, the trapping time of orbits in the former category will be about twice as long as those in the latter category.

Near any hyperbolic point there exist coordinates $(\xi, \eta)$ in which the mapping may be written as

$$
\xi_{t}=\lambda \xi_{t-1}, \quad \eta_{t}=\lambda^{-1} \eta_{t-1}
$$

where $\lambda$ is the Lyapunov number at the fixed point. The trajectories lie on hyperbolae of the form $\xi \eta= \pm \xi_{0}^{2}$. Since the island encircling trajectories dominate the long-time behavior of $f_{t}$, we define the trapping time $t\left(\xi_{0}\right)$ as the twice the time it take to get from $\eta=1$ to $\xi=1$. (The factor of two accounts for the two encounters with the fixed point.) We find

$$
t\left(\xi_{0}\right)=-4 \log \xi_{0} / \log \lambda, \quad d t\left(\xi_{0}\right) / d \xi_{0}=-4 /\left(\xi_{0} \log \lambda\right) .
$$

The area $A\left(\xi_{0}\right)$ between the hyperbola, the axes, and the lines $\xi=1$ and $\eta=1$ is

$$
A\left(\xi_{0}\right)=\xi_{0}^{2}\left(1-2 \log \xi_{0}\right)
$$

The trapping statistics are then given by

$$
f_{t} \sim \frac{1}{t} \frac{d A}{d t} \sim \exp \left(-\frac{1}{2} \log \lambda t\right)
$$

For the fixed point at $(0, \sqrt{K})$,

$$
\lambda=1+2 \sqrt{K}+2 \sqrt{\sqrt{K}+K} \approx 1+2 K^{1 / 4}, \quad f_{t} \sim \exp \left(-K^{1 / 4} t\right) .
$$

$C_{\tau}$ behaves in the same way. For $K=10^{-4}$, the decay rate should be about 0.1 , which is indeed what was observed in fig. 3(c). Similar agreement is seen at $K=10^{-3}$ and $10^{-2}$. However at $K=0.1$, the central island has shed a chain of sixth-order islands and the foregoing analysis does not apply. This case is discussed in the next section. 


\section{The results for $K=0.1$.}

We have measured $f_{t}$ for $K$ between 0 and 1.3 at intervals of 0.05 , and at most of the values of $K$ a slow algebraic decay of $f_{t}$ is seen. A representative case is $K=0.1$, whose trapping statistics are given in fig. 6(a), which illustrates the slow decay for very long times $t \sim 10^{7}$. Also given in fig. 6 are $P_{t}, C_{\tau}$, and $\alpha \equiv-d \log C_{\tau} / d \log \tau$ (thus locally $C_{\tau} \sim \tau^{-\alpha}$ ). This last plot shows the power at which $C_{\tau}$ decays varying between about $\frac{1}{4}$ and $\frac{3}{2}$.

A glance at fig. 2 shows the origin of this behavior. The central island is surrounded by a chain of sixth-order islands. Around each of these islands are several other sets of islands. This picture repeats itself at deeper and deeper levels. A particle which manages to penetrate into this maze can get stuck in it for a long time.

For $\tau \lesssim 10^{4}$, fig. $6(\mathrm{~d})$ gives $\alpha \approx \frac{1}{4}$. Correspondingly we have $P_{t} \sim t^{-p}$ where $p=$ $1+\alpha \approx 5 / 4$. This is close to the asymptotic $(t \rightarrow \infty)$ result found in ref. 5 for the whisker map, in which $\langle p\rangle \approx 1.45$. This is another indication that the behavior of a Hamiltonian with a divided phase space has "universal" properties. However, in our case, $\alpha$ shows some strong variations beyond $\tau \approx 10^{4}$ where $C_{\tau}$ "steps down" (e.g., between $10^{4}$ and $3 \times 10^{5}$ ). This means that the asymptotic form of $C_{\tau}$ is very difficult to determine numerically.

The diffusion coefficient $D$ is given by the summation of $C$ and is approximately $6400 \pm$ 800. The error is estimated by calculating $D$ separately for subsets of the orbits sampled. Unfortunately, because a few very long orbit segments have such a large effect on $D$, the individual observations of $D$ come from a highly skewed distribution and the error may be severely underestimated. We will try to get a idea of the error by asking what behavior is possible for $f_{t}$ for $t_{a}=10^{8}<t<2 \times 10^{9}=t_{b}$ ( $t_{b}$ is the length of the orbits used to compute $f_{t}$ in fig. 6). Since no segments were observed in this range, we have

$$
\int_{t_{a}}^{t_{b}} M\left(t_{b}-t\right) f_{t} d t \lesssim 1
$$

where $M=1600$ is the number of orbits used. This just says that the expected number of orbit segments in this range of $t$ is less than 1 (see appendix A). Suppose that $f_{t}=$ $A\left(t / t_{a}\right)^{-(2+\alpha)}$ where $\alpha$ is the exponent at which $C_{\tau}$ decays and $A$ is the value of $f_{t}$ at $t=t_{a}=10^{8}$. For $t_{b} \gg t_{a}$ and $\alpha>0$, we can evaluate the integral to give $M A t_{a} t_{b} /(1+\alpha)$ approximately. If we take $A=10^{-20}$ (this value was estimated from fig. 6(a)), we find $\alpha \gtrsim 2$. In the case of the slowest decay, $\alpha=2$, the portion of $f_{t}$ between $t_{a}$ and $\infty$ would increase $D$ by about a factor of 2 over the value given above. If $f_{t}$ takes another step down near $t=10^{8}$, then $A$ might be smaller and smaller values of $\alpha$ would be possible and the maximum error in $D$ would be larger. For instance with $A=0.3 \times 10^{-20}$, then all values of $\alpha<0$ are consistent with the numerical observations. Since $C_{\tau}$ sums to infinity for all $\alpha \leq 1, D$ may well be infinite!

If $D$ is indeed infinite, we would wish to know how a group of particles spreads with time. We again consider the drunkard's walk based on $Q^{*}$ which was introduced in section 3 . The second moment of $r$ is related to the correlation function by

$$
S_{t} \equiv\left\langle\left(r_{t}-r_{0}\right)^{2}\right\rangle=t C_{0}+2 \sum_{\tau=1}^{t}(t-\tau) C_{\tau} .
$$

This is plotted in fig. 7 (a), using the data of fig. 6 . For $t \lesssim 10^{4}, S_{t}$ grows somewhat faster than $t^{3 / 2}$ (see fig. $7(\mathrm{~b})$ ) and even until $t \approx 10^{7}, S_{t}$ is growing significantly faster than linearly. Beyond $10^{7}$, the numerical data shows a convergence to a linear rate; but this is 
merely because no segments longer than about $6 \times 10^{7}$ were observed. For $t \rightarrow \infty, S_{t}$ grows as $t^{2-\alpha}$, assuming that the exponent $\alpha$ at which $C_{\tau}$ decays asymptotically is less than 1 . If the diffusion coefficient is estimated from $D_{t}=\frac{1}{2} S_{t} / t$, then $D_{t}$ grows with $t$ as shown in fig. $7(\mathrm{c})$.

When applying these results to the general mapping, we will need to know the area $b_{2}$ occupied by the stochastic trajectories (the dark area in fig. 2). This was calculated by dividing the phase space into $1024 \times 1024$ little boxes, iterating the mapping many times, and counting the number of occupied boxes. It is important iterate the map enough times so that (a) the expected occupation number of each box is reasonably large and (b) the trajectories have time to wander into all the nooks and crannies. (In practice, the second requirement is more stringent.) With $x_{\min }, x_{\max }$, and $X_{r}$ as given in the caption to fig. 6 , the area of the stochastic component is found to be about $b_{2}=0.693$.

\section{Application of the results.}

We wish now to determine the contribution of an island to the correlation function of an orbit in the stochastic component of phase space of a general two-dimensional areapreserving mapping $G$. (The analysis applies equally well to Hamiltonians with two degrees of freedom. The phase space is then the Poincaré surface of section and the unit of time is the period of the island.)

For simplicity we begin by considering the case where there is a single small island embedded in the connected stochastic component. Let the total area occupied by the stochastic component be $A_{1}$. Suppose a small island centered at $\mathbf{x}_{0}$ is immersed in the stochastic sea. When iterating $Q^{*}$, we have been approximating $G$ in a small region around $\mathbf{x}_{0}$. The square defined by $x_{\min }$ and $x_{\max }$ in $Q^{*}$ is transformed into a small box (parallelogram) of area $B_{0}$. The ratio of the areas in the two spaces is $B_{0} / L^{2}=\gamma$. Suppose the area of the stochastic component of $G$ which lies inside this box is $B_{1}$. (In the notation of appendix A, $B_{1}=\gamma b_{1}$.) Recall that $Q^{*}$ also contains spurious islands (accelerator modes) which have no counterpart in $G$. To account for these islands we define $f_{t}^{*}$ as in (A1). From this we can derive $\sum f_{t}^{*}=1 / \alpha^{*}$ and $p_{t}^{*}=\alpha^{*} f_{t}^{*}$ (parallelling the definitions made in section 3 ).

It is useful to begin by forming an idea of what a stochastic trajectory will look like. Orbits will consist of alternating trapped and free segments. The trapped segments are those which are restricted to $B_{1}$, while the free segments are those excluded from $B_{1}$. (Here and in the following we use $B_{1}$, etc., to refer to a particular subset of phase space as well as the area of this subset.) The basic assumption is that each visit to the island is uncorrelated with the previous one. So, on first entering the area $B_{1}$, we assume that a segment of length $t$ will be chosen randomly with probability $p_{t}^{*}$. A simple model for the motion in the stochastic region $A_{1}-B_{1}$, which excludes the region near the island, is as follows. The first point after a trapped segment is randomly (and with a uniform distribution) situated in the $A_{1}-B_{1}$. This is in accord with the picture that once an orbit leaves $B_{1}$, it rushes away from the vicinity of $B_{1}$ extremely quickly. If this point is the pre-image under $G$ of $B_{1}$, then the next point is the first point of another trapped segment. Otherwise another point is chosen at random in $A_{1}-B_{1}$ and the procedure is repeated. The mean length of trajectories trapped in $B_{1}$ is $\alpha^{*}$. The area of the points which are initial points of trapped segments is therefore $B_{1} / \alpha^{*}$. These are the points whose pre-images lie outside $B_{1}$. Thus the probability that a point in $A_{1}-B_{1}$ is a pre-image of $B_{1}$ is $\epsilon=\left(B_{1} / \alpha^{*}\right) /\left(A_{1}-B_{1}\right)$. The probability that a

particular free segment has length $t$ is then $\epsilon(1-\epsilon)^{t-1}$. These probabilities ensure that ratio 
of time that the trajectory spends in $B_{1}$ and in $A_{1}-B_{1}$ is in the ratio of the area of these regions. (The assumptions made to obtain the distribution of lengths of the free segments is probably overly restrictive. However such a "memory-less" model is probably accurate for the long times we are interested in.) This model is discussed in more detail in appendix $\mathrm{C}$ where it is used as a basis for constructing a Markov-chain approximation of the motion.

Consider the correlation function

$$
\mathcal{C}(\tau)=\langle h(\mathbf{x}(t)) h(\mathbf{x}(t+\tau))\rangle_{t},
$$

where $h$ is some smooth function of the position in phase space $\mathbf{x}$ (in particular we require that it is a constant throughout $\left.B_{1}\right)$. We shall assume that the mean value of $h(\mathbf{x}(t))$ is zero for the stochastic orbits. We identify those terms in (13) for which $\mathbf{x}(t)$ and $\mathbf{x}(t+\tau)$ belong to the same trapped segment as $\mathcal{C}_{\text {is }}(\tau)$ the contribution to $\mathcal{C}(\tau)$ due to the island. (Thus for such terms we have $\mathbf{x}\left(t+\tau^{\prime}\right) \in B_{1}$ for all $\tau^{\prime}$ such that $0 \leq \tau^{\prime} \leq \tau$.) Except for $\tau=0$, the other terms are smaller by a factor of about $B_{1} / A_{1}$, which is typically very small. This is so because $h$ has a zero mean and because of the rapid mixing of orbits in $A_{1}-B_{1}$. This question is examined in appendix $\mathrm{C}$ where it is also shown that the additional terms do not contribute to the diffusion coefficient. $\mathcal{C}_{\text {is }}(\tau)$ may be written as

$$
\mathcal{C}_{\text {is }}(\tau)=h^{2}\left(\mathbf{x}_{0}\right) \frac{B_{1}}{A_{1}} \sum_{t=\tau}^{\infty}(t-\tau) f_{t}^{*} .
$$

The first factor arises because both endpoints are in $B_{1}$ and $h(\mathbf{x}) \approx h\left(\mathbf{x}_{0}\right)$ for such points. The second factor is the probability that $\mathbf{x}(t)$ lies in $B_{1}$, and the sum is the probability that $\mathbf{x}(t+\tau)$ belongs to the same trapped segment as $\mathbf{x}(t)$. This sum is just the correlation function defined in terms of $f_{t}^{*}$ instead of $f_{t}$. For large $\tau$, we can substitute for $f_{t}^{*}$ using (A2), and the sum becomes $\left(b_{2} / b_{1}\right) C_{\tau}$. Thus the contribution to the correlation function due to the island is

$$
\mathcal{C}_{\text {is }}(\tau)=h^{2}\left(\mathbf{x}_{0}\right) \frac{\gamma}{A_{1}} b_{2} C_{\tau} .
$$

Equation (A3) shows that this result is independent (for large $\tau$ ) of the choice of $x_{\max }$ and $x_{\min }$ (which is as it should be).

If $h$ is the rate of change of one of the components of $\mathbf{x}$, e.g., $h(\mathbf{x})=d x / d t$, then the island enhances the $x$-space diffusion coefficient by

$$
\mathcal{D}_{\text {is }}=h^{2}\left(\mathbf{x}_{0}\right) \frac{\gamma}{A_{1}} b_{2} D,
$$

where $D$ (assuming that it exists) is given in (6). Whether or not $D$ exists, the mean squared $x$ position of a group of particles initially concentrated in a small region is enhanced by

$$
\mathcal{S}_{\text {is }}(t)=h^{2}\left(\mathbf{x}_{0}\right) \frac{\gamma}{A_{1}} b_{2} S_{t},
$$

where $S_{t}$ is given by (12).

If there are more than one island, then the contributions should be added together in $\mathcal{C}_{\tau}$ and $\mathcal{D}$. If there is a chain of $N$ th order islands at $\mathbf{x}_{0}, \mathbf{x}_{1}, \ldots, \mathbf{x}_{N}=\mathbf{x}_{0}$, their contribution to the correlation function $\mathcal{C}(N \tau+j)$ is

$$
\mathcal{C}_{\text {is }}(N \tau+j)=\sum_{i=0}^{N-1} h\left(\mathbf{x}_{i}\right) h\left(\mathbf{x}_{i+j}\right) \frac{\gamma}{A_{1}} b_{2} C_{\tau},
$$


where $\gamma=B_{0} / L^{2}$ for one of the islands and $0 \leq j<N$. The contribution to $\mathcal{D}$ (assuming again that it exists) is

$$
\mathcal{D}_{\text {is }}=\left(\sum_{i=0}^{N-1} h\left(\mathbf{x}_{i}\right)\right)^{2} \frac{\gamma}{A_{1}} b_{2} D
$$

and similarly for $\mathcal{S}_{\text {is }}(t)$

Let us apply these results to the first-order accelerator modes for the standard mapping (1). These are first-order islands which appear at $k=2 \pi n$ ( $n$ an integer) [1]. The acceleration in such a mode is $r_{t}-r_{t-1}= \pm 2 \pi n$. These modes appear in pairs, one with each sign of the acceleration. We will take the area of the stochastic component $A_{1}$ to be equal to the entire area of phase space $(2 \pi)^{2}$. The relation between the parameters is found by matching the residues at the stable fixed point. This gives $k^{2}=(2 \pi n)^{2}+16 K$. When transforming to the quadratic map $Q$, lengths are magnified by a factor $\frac{1}{2} \pi n[3]$ and so $\gamma=(2 / \pi n)^{2}$. If we wish to estimate the diffusion coefficient, we must define $h$ to be the acceleration; thus $h\left(\mathbf{x}_{0}\right)= \pm 2 \pi n$. For $K=0.1$, we take $b_{2}=0.693$ and the proportionality constant connecting the scripted and unscripted quantities above is $2 h^{2}\left(\mathbf{x}_{0}\right)\left(\gamma / A_{1}\right) b_{2} \approx 0.56$. (The factor of 2 accounts for the presence of the two islands.)

Using $\mathcal{D}_{\mathrm{ql}}=(\pi n)^{2}$ and taking 6400 as a lower bound for $D$, we find that the contribution to the diffusion coefficient is increased over its quasi-linear value by a factor of at least $360 / n^{2}$. Thus for $n=1$ or $k \approx 6.41$, the islands completely dominate the diffusion. The first-order accelerator modes continue to have such a large effect at least until $k \approx 100$. If $D$ is in fact infinite, even arbitrarily small accelerator modes will eventually dominate the motion and fig. 7 can be used to estimate the time at which the accelerator modes become important.

\section{Discussion.}

We have looked at the effect of a small island on the correlation function for the stochastic trajectories of Hamiltonians of two degrees of freedom. When the parameter $K$ is small, the problem may be treated analytically and we find that the contribution to the correlation function $C_{\tau}$ is zero for $\tau \gtrsim 5.1454|K|^{-1 / 4}$ when $K<0$, decays as $\tau^{-5}$ when $K=0$, and decays as $\exp \left(-K^{-1 / 4} \tau\right)$ for $K>0$.

The more interesting case is when $K$ is not small and the island is surrounded by other islands. In the case we considered in detail $K=0.1$, the decay of the correlation function is algebraic and very slow (roughly as $\tau^{-1}$ ) out to times on the order of $\tau \sim 10^{7}$. Even when the islands are small, this can still lead to an enormous increases of quantities such as the diffusion coefficient. Although it has not been definitely established, there are strong indications that the diffusion coefficient may be infinite, indicating that the distribution of particles does not obey a diffusion equation and that the particles spread more rapidly than diffusively. Such behavior is fairly typical, having been observed at several other values of $K$.

It would be interesting to know how the distribution of particles does evolve in time. If we consider a system like the standard mapping at a parameter value for which an accelerator mode exists, then for large $t$ this distribution could, in principle, be found from $f_{t}$, but its determination is beyond the scope of this work. For now, we observe that the distribution will be far from Gaussian for times at least until $t=10^{8}$. It will contain a very small but extremely long tail that contributes significantly to the variance. This has important 
consequences for numerical experiments. Imagine trying to measure $S_{t}$ for $t=10^{8}$ by directly measuring $r_{t}-r_{0}$ for $N$ particles. If $N$ is merely some "reasonably" large number like 1000 then $S_{t}$ will most likely be greatly underestimated. (To counteract this there is a tiny probability that $S_{t}$ will be fabulously overestimated.) We know that we should sample some orbits with $r_{t}-r_{0} \sim 10^{7}$ to be able to estimate $S_{t}$ accurately. But since $S_{t} \sim 10^{4}$, we need to sample at least $\left(10^{7}\right)^{2} / 10^{4}=10^{10}$ orbits before the effect of such a long segment is correctly diluted. Obviously such a calculation is totally out of the question. A much better approach is to measure the correlation function and to derive $S_{t}$ using (12). On the other hand, the requisite number of orbits probably are sampled in real experiments. For instance in plasma physics applications the total number of orbits is typically $10^{14}$.

There are several questions still to be answered. What is the long-time behavior of the correlation function? Figure 6 shows that it has not attained any well-defined asymptotic limit by $\tau=10^{7}$. What determines the asymptotic behavior of the correlation function? The simplest picture we can form for treating this problem would go something like this: There is some outer KAM curve marking the boundary of the main island centered at $(-\sqrt{K}, 0)$. According to Greene [11], this curve is approximated from the outside by a sequence of islands whose winding numbers are the rational approximants to the irrational winding number of the KAM curve. Thus the long time behavior of $f_{t}$ may be found by considering how an orbit wanders through these islands to approach the KAM curve. Greene [12] found an algebraic decay of the correlation function based on a simple model of such a process. Equivalently this behavior may be obtained from a diffusion equation in which the diffusion coefficient approaches zero sufficiently fast as the KAM curve is approached. This picture was the one proposed in ref. 5 .

Such pictures are however probably incomplete. There is no reason to suppose that the KAM curve around the central island determines the asymptotic behavior of $f_{t}$. For $K=0.1$, it could equally well be the last KAM curve around the sixth-order islands which surround the central island, or the KAM curve around one of the chains of islands around the sixth-order islands, or all of them together! For instance, the longest orbit segment seen for $K=0.1$, whose length was about $6 \times 10^{7}$, spends most of its time around such a chain of islands whose order is $6 \times 23=138$. (One of the members of this chain is visible in fig. 2(b) at $x+\sqrt{K} \approx 0.352$ and $y \approx 0.034$. An enlargement of this long orbit is shown in fig. 8.) Is it the KAM surface around this island chain that will determine the asymptotic behavior of $f_{t}$ ? In this picture, the asymptotic behavior is determined by the islands at a finite depth in the islands-around-islands hierarchy. This may be false. Perhaps as $t$ is increased, we must look at deeper and deeper levels of the hierarchy. Such is the view taken by Chirikov in ref. 13. We may also have to jump between branches of the hierarchy as $t$ increases. (This is supported by the observation that the three longest orbits seen for $K=0.1$ are each stuck close to different island chains.)

The absence of any idea as to the asymptotic behavior of $C_{\tau}$ makes it virtually impossible either to obtain numerically an upper bound on the diffusion coefficient or to establish that it is unbounded. Such questions can probably only be answered when this problem is better understood analytically. In any case, it may be necessary to take a critical look at extraneous effects which act as an extrinsic noise source and which will cut off the very long correlations.

Finally, what is a measure of these islands? This gives the overall importance of this phenomenon. We already have Sinai's estimate as to the number of islands that will be created. What is still needed is some estimate of their size and of the interval in parameter 
space in which they exist. Numerical evidence suggests that these both decrease rapidly with the period. The latter quantity would enable us to say what is the measure of parameter space on which we expect to see islands.

\section{Acknowledgments}

This work was supported by the U.S. Department of Energy under Contract DE-AC0276-CHO3073. I began it while at the Institute of Plasma Physics, Nagoya University, participating in the U.S.-Japan Fusion Cooperation Program. Some of the work was carried out while at the Aspen Center for Physics. I would like to thank J. M. Greene, R. S. MacKay, and F. Vivaldi for stimulating discussions. B. V. Chirikov provided some enlightening comments on a preliminary version of this paper.

\section{Appendix A: Properties of the periodic mapping.}

In order to obtain correct trapping statistics, we would like to uniformly sample the trajectories the area outside the islands of $Q$ and measure how long each trajectory spends close to the islands. We achieve this goal by looking at long trajectories of $Q^{*}$. In this case, "close" is determined by $x_{\min }$ and $x_{\max }$. However, fig. 2 shows that the area outside the islands is not sampled uniformly. There are holes in it corresponding to the spurious islands. Suppose that the order of a particular set of islands is $n$. Particles in this set of islands will have looped around the torus at least once in the $x$ or $y$ direction after $n$ iterations of $Q^{*}$. (These islands are accelerator modes.) A trajectory of length $n$ within these islands is made up of one or more segments. It cannot be part of a longer segment. By omitting the trajectories within the islands we are therefore undercounting the segments with lengths less than or equal to $n$.

We can express this in a more quantitative way. The total area of phase space in $Q^{*}$ is $b_{0}=L^{2}$. We define $b_{1}$ to be that part of $b_{0}$ which is not occupied by the islands of $Q$ and $b_{2}$ to be the area of the stochastic component of $Q^{*}$, i.e., that part of $b_{0}$ which is not occupied by the islands of $Q^{*}$. The difference between these two, $b_{1}-b_{2}$ gives the area of the spurious islands. To include the effect of the spurious islands, we define $f_{t}^{*}$ by

$$
f_{t}^{*}=\frac{b_{2}}{b_{1}} f_{t}+\frac{b_{1}-b_{2}}{b_{1}} g_{t},
$$

where $g_{t}$ is the contribution from the regions of phase space occupied by the spurious islands. If $g_{t}$ is normalized like $f_{t}$ to have a first moment of unity, then $f_{t}^{*}$ has the same normalization. The factors multiplying $f_{t}$ and $g_{t}$ are just the relative areas of those regions of phase to which these trapping statistics apply. Now $g_{t}$ specifies the lengths of orbit segments in the spurious islands, which must be less than or equal to $n_{\max }$ the maximum period length for these islands. Thus $g_{t}$ is zero for $t>n_{\max }$. If we wish to calculate $C_{\tau}$, we need only know the trapping statistics for $t>\tau$. So as long as $\tau>n_{\max }$, we may ignore $g_{t}$ and use

$$
f_{t}^{*}=\frac{b_{2}}{b_{1}} f_{t} .
$$

These spurious islands have one other interesting effect. They introduce correlations between the lengths of successive segments. Consider an orbit close to such an island chain of period 3. Then, in the simplest case, we expect to see a succession of segments of length 3. Such correlations are obviously a peculiarity of $Q^{*}$. In the general mapping, where the 
particle spends some time in the stochastic sea before returning to the neighborhood covered by $Q^{*}$, the trapped segments will be sampled randomly.

We next examine the effect of the definition of "close," i.e., at the effect of changing $x_{\min }$ and $x_{\max }$. Consider the effect of increasing the box size by decreasing $x_{\min }$ and increasing $x_{\max }$. This has two effects: new segments which never entered the original box appear; and all the original segments may be extended both forwards and backwards. As long as the original box was large enough, the new segments will all be short (since they never get close to the islands). By the same argument, the extension of the orbit segments will be small. Because of the extremely rapid departure of orbits away from the islands, we can, for instance double, the edge of the box and only increase the average length of the orbits segments by less than one. So the main effect of increasing the box size will be to increase the number of short segments. As in the case of the accelerator modes, we can quantify this. If we change $x_{\min }$ to $x_{\min }^{\prime}$ and $x_{\max }$ to $x_{\max }^{\prime}$, then, for large enough $t$, the new trapping statistics $f_{t}^{\prime}$ are given by

$$
b_{2}^{\prime} f_{t}^{\prime}=b_{2} f_{t},
$$

where $b_{2}^{\prime}$ is the area of the stochastic component in the new box.

Lastly, we address the problem of estimating the trapping statistics from an orbit of length $T$. Consider an infinitely long time record consisting of segments of various lengths $t=1,2, \ldots$. Pick a random record of length $T$ and call $N_{t}$ the expected number of segments of length $t$. (We do not count partial segments at the ends.) Then

$$
N_{t}= \begin{cases}0 & \text { for } t>T \\ (T+1-t) f_{t} & \text { for } 0<t \leq T\end{cases}
$$

This may be proved by induction. When $T=0$, we have $N_{t}=0$ as required. When we extend the record from $T-1$ to $T$, the only way a new segment of length $t$ can be included is if the particle is at the end of such a segment at time $T$. Because the probability of this happening is $f_{t}, N_{t}$ increases by $f_{t}$ for $t \leq T$.

Thus we divide actual number of orbit segments by $(T+1-t)$ to obtain an unbiased estimate of the trapping statistics, $f_{t}$. The proof given above does not depend on the segments appearing in a random order. This is important because, as we have seen, there will be correlations between successive segments in $Q^{*}$. One problem with the method we use for measuring $N_{t}$ is that the beginning of the record is not arbitrary, because initially we have $(X, Y)$ uniformly distributed on the line $X=0$. If $T$ is large, this is not expected to affect the results very much.

\section{Appendix B: Numerical methods used.}

In order to make the reduction of $x$ and $y$ in $Q^{*}$ to their base intervals as cheap as possible, the $L \times L$ square is reduced to a unit square with a zero origin. Thus new coordinates $X$ and $Y$ are defined by

$$
x=x_{\min }+L X, \quad y=-\frac{1}{2} L+L Y .
$$

In these coordinates $Q^{*}$ becomes

$$
N: \quad Y_{t}=Y_{t-1}+G\left(X_{t-1}\right) \quad(\bmod 1), \quad X_{t}=X_{t-1}+Y_{t}-\frac{1}{2} \quad(\bmod 1),
$$

where

$$
G(X)=a X^{2}-b X+c, \quad a=2 L, \quad b=-4 x_{\min }, \quad c=2\left(x_{\min }^{2}-K\right) / L .
$$


We will always choose $x_{\min }<-\sqrt{K}$, so that the stable fixed point at $(x, y)=(-\sqrt{K}, 0)$ is included in the base intervals. Thus the quantities $a, b$, and $c$ are all positive.

$N$ is implemented using fixed-point arithmetic on the Cray-1. This should be compared with the use of integer mappings by Rannou [14]. In both cases "exact" (in a sense to be defined later) numerical mappings can be defined. The principal difference is in the coarseness of the grid on which the mapping is defined. The finest grid that Rannou used was $800 \times 800$. In contrast, our grid is about $2^{48} \times 2^{48}$, so that its resolution is close to that obtained with floating point numbers. The use of fixed-point arithmetic also results in faster performance because the extraction of the integer and fractional parts of a number just correspond to masking operations.

First we define the notation for fixed-point numbers. We view the full 64-bit word as representing a number in twos-complement binary notation with the "binary" point placed before the last 48 bits. Addition and subtraction are performed by the integer add and subtract instructions. Multiplication of fixed-point numbers is accomplished by the floatingpoint multiply instruction provided the two numbers lie in the range $[0,1)$. Multiplication of a number by $2^{m}$ where $m$ is a non-negative integer is accomplished by shifting the word to the left $m$ places (with zeros introduced on the right).

The function $G$ is then computed by writing it as

$$
G(X)=2^{m}\left(A X^{2}-B X+C\right), \quad(A, B, C)=2^{-m}(a, b, c)
$$

where $m$ is the smallest non-negative integer such that $A, B$, and $C$ all lie in $[0,1) . X$ also lies in this range so that all the multiplications in $G$ may be carried out by computing $A X^{2}-B X+C$ with the add, subtract, and multiply instructions and shifting the result $m$ places to the left. Since the low $m$ bits of $G$ are always zero, then, assuming that the low $m$ bits of both $X$ and $Y$ are initially zero, they will remain zero. Thus there will be $n=\left(2^{48-m}\right)^{2}$ points accessible in the unit square. (Typically $m=2$ and $n \approx 10^{27}$.)

We are now ready to give the procedure for gathering the trapping statistics $f_{t}$.

(1) Read in $K, x_{\min }, x_{\max }, X_{r}, T, q$. (The trapping statistics will be collected for the $q$ th power of the mapping. $T$ is the total number of iterations of $N^{-q} . X_{r}$ defines the size of the randomizing zone.)

(2) Initialize $B_{i} \leftarrow 0$ for $0<i \leq 150, t \leftarrow 0, \tau \leftarrow T,(X, Y) \leftarrow(0,0)$. Initialize the random number generator with a "random" seed. ( $B_{i}$ are the bins used for collected the trapping statistics. The counter $t$ gives the length of the orbit segment so far. The number of steps still to do is given by $\tau$.)

(3) Set $L \leftarrow x_{\max }-x_{\min }, a \leftarrow 2 L, b \leftarrow-4 x_{\min }, c \leftarrow 2\left(x_{\min }^{2}-K\right) / L$. Calculate $m, A, B, C$, according to the the prescription given above.

(4) Set $\tau \leftarrow \tau-1$. If $\tau<0$ stop.

(5) Initialize $P \leftarrow$ false. ( $P$ is set to true when the trajectory leaves the base square.) Set $t \leftarrow t+1$

(6) Set $(X, Y) \leftarrow R(X, Y)$ where the mapping $R$ is given below.

(7) Set $(X, Y) \leftarrow N^{-q}(X, Y)$ where the mapping $N^{-q}$ is given by iterating the mapping $N^{-1}$ given below $q$ times.

(8) If $P$ is false go to step 4.

(9) Record new orbit segment by setting $i \leftarrow b(t), B_{i} \leftarrow B_{i}+1$. Re-initialize $t \leftarrow 0$. Go to step 4.

The mapping $R$ is defined by 
(6.1) If $X>X_{r}$ return.

(6.2) Set $P \leftarrow$ true, $Y \leftarrow Y+U$ where $U$ is a uniformly distributed random variable $(\bmod 1)$. Return.

The mapping $N^{-1}$ is defined by

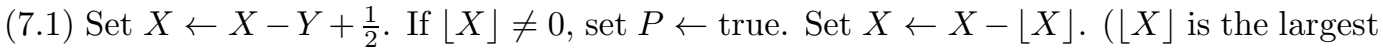
integer satisfying $X \geq\lfloor X\rfloor$.)

(7.2) Set $Y \leftarrow Y-G(X)$ where $G(X)$ is calculated as outlined above. If $\lfloor Y\rfloor \neq 0$, set $P \leftarrow$ true. Set $Y \leftarrow Y-\lfloor Y\rfloor$. Return.

The bin number $b(t)$ is defined by

$$
b(t)= \begin{cases}t & \text { for } t \leq 50 \\ \left\lfloor 30 \log _{10} t\right\rfloor & \text { for } 50<t<10^{5} \\ 150 & \text { for } 10^{5} \leq t\end{cases}
$$

Finally the trapping statistics $f_{t}$ are obtained by

$$
F_{i}= \begin{cases}B_{i} /(T+1-i) & \text { for } i \leq 50, \\ \frac{B_{i} /\left(T+1-10^{(i+1 / 2) / 30}\right)}{\left\lceil 10^{(i+1) / 30}\right\rceil-\left\lceil 10^{i / 30}\right\rceil} & \text { otherwise, }\end{cases}
$$

together with

$$
f_{t}=F_{i} / q^{2},
$$

where $i=b(\lfloor t / q\rfloor)$.

The mapping $R$ randomizes the $Y$ positions of the particles in a thin zone $\left(0 \leq X \leq X_{r}\right)$ on the left edge of the square. ( $X_{r}$ is chosen to be small, typically 0.02.) As long as the randomizing zone is sufficiently far removed from the islands, $R$ has no effect on the length of orbits which are trapped for moderately long times. $R$ serves two purposes. When it is first applied, it picks a random initial condition on the line $X=0$. It also stops the trajectory from being periodic either because of the spurious accelerator modes or because a stochastic trajectory has eventually returned to its starting position. This results in a more rapid sampling of phase space and so better statistics are obtained.

Since the accumulation of the statistics $B_{i}$ is quite expensive compared with iterating the map, we look instead at the $q$ th power of the mapping. We count the orbit segment as having ended if the orbit wrapped around the torus in any of the $q$ iterations. This leads to some uncertainty in $f_{t}$ for $t \sim q$. However, we are most interested in very long trapping times for which $t \gg q$.

Full advantage is taken of the vectorization capabilities of the Cray- 1 by following $M$ (a multiple of 64) orbits and averaging the results. Finally, the critical parts of the algorithm were handed-coded in CAL, the Cray Assembly Language. The execution time is approximately $(360+73 q) T$ ns per particle. This is about 6 times faster than a straightforward implementation in FORTRAN using floating-point arithmetic. The most time-consuming computations were those of fig. 6 where $M=1600$ orbits of length $q T=2 \times 10^{9}$ were used, a total of $3.2 \times 10^{12}$ iterations of $N$. This consumed about 65 hours of CPU time on the Cray-1.

Aside from the computation of $G(X)$, all the calculations are exact. $N$ is therefore areapreserving, or, since only a discrete set of numbers may be represented on the computer, it is better to say that it is one-to-one. A trajectory may be run backwards by

$$
N^{-t}=J^{-1} N^{t} J
$$


where $J$ is the involution ( $J^{2}$ is the identity)

$$
J: \quad X_{t}=X_{t-1}-Y_{t-1}+\frac{1}{2} \quad(\bmod 1), \quad Y_{t}=-Y_{t-1} \quad(\bmod 1)
$$

which may be calculated exactly using fixed-point arithmetic.

Because of the discreteness of the number system on a computer, all the trajectories of a numerical mapping are eventually periodic. Because $N$ is a one-to-one mapping, the whole trajectory will be periodic. There will be no initial transient. Presumably the typical period length of an orbit in the stochastic part of phase space is very long. Rannou [14] gives $\frac{1}{2}(n+1)$ as the average length of a trajectory in a random permutation of $n$ points. (This is to be compared with the period length for an orbit in a random function on $n$ points which is $\sqrt{\pi n / 8}+\frac{1}{3}$ approximately [15]. The distinction between a function and a permutation is that a permutation is a one-to-one mapping of the $n$ points onto themselves, while a function is a many-to-one mapping of the points into themselves. It is not clear whether the stochastic orbits of floating-point mappings are closer to those of random permutations or random functions.) However, $N$ is not a random mapping because it possesses symmetries. In the case of the standard mapping this reduces the expected length of stochastic orbits to $O(\sqrt{n})$ [14]. Although this number is still large (about $10^{13}$ ), we expect there to be a large number of trajectories with shorter periods. Examples of such trajectories are those in the accelerator modes which are restricted to a small fraction of phase space. By composing the mapping with $R$, we give the trajectory a random step whenever it returns to its starting point (and usually before that). We therefore ensure that a particle stays in these short periodic orbits for at most $q$ periods. (Of course the random number generator is an example of a mapping. But it is specially chosen to have a very long period of $2^{40}$, so the random number generator repeats itself after about $10^{12} q$ iterations of $N$.)

\section{Appendix C: Correlation function for Markov model.}

In section 6 , we presented a model for the motion of a stochastic trajectory when an island is present. We wish to make that model more precise so that we can accurately assess the influence of the island on the correlation function. We do this by modeling phase space with a finite number of points and writing down a Markov transition matrix $\mathbf{P}$ for evolution of the distribution function.

Suppose there are a total of $N$ points. In the simplest case, there is just one trapped segment of length $m$. The stochastic sea consists of the other $n=N-m$ points, one of which is special in that it is the pre-image of one of the trapped points. For example if $N=7, m=3, n=4$, then $\mathbf{P}$ is given by

$$
\mathbf{P}=\left(\begin{array}{ccccccc}
0 & 0 & 0 & 0 & 0 & 0 & 1 \\
1 & 0 & 0 & 0 & 0 & 0 & 0 \\
0 & 1 & 0 & 0 & 0 & 0 & 0 \\
0 & 0 & \frac{1}{4} & \frac{1}{4} & \frac{1}{4} & \frac{1}{4} & 0 \\
0 & 0 & \frac{1}{4} & \frac{1}{4} & \frac{1}{4} & \frac{1}{4} & 0 \\
0 & 0 & \frac{1}{4} & \frac{1}{4} & \frac{1}{4} & \frac{1}{4} & 0 \\
0 & 0 & \frac{1}{4} & \frac{1}{4} & \frac{1}{4} & \frac{1}{4} & 0
\end{array}\right) .
$$

The entry in the $j$ th row and $i$ th column is the probability that a particle at point $i$ at time $t$ is at point $j$ at time $t+1$. Thus we see that points $1-3$ constitute the trapped segment, 
while points $4-8$ are the stochastic points with point 8 being the pre-image of one of the trapped points.

In the more general case, there may be $M$ trapped segments, whose lengths are $m_{k}$ for $1 \leq k \leq M$. Suppose the members of the $k$ th trapped segment are $T_{k}=\left\{p_{k}^{(1)}, p_{k}^{(2)}, \ldots\right.$, $\left.p_{k}^{\left(m_{k}\right)}\right\}$, and that the pre-image of $p_{k}^{(1)}$ is $q_{k}$. We will also define $T_{k}^{\prime}=\left\{q_{k}\right\} \cup T_{k}, T=\bigcup_{k} T_{k}$, $T^{\prime}=\bigcup_{k} T_{k}^{\prime}, S=T^{\mathrm{c}}, S^{\prime}=T^{\prime c}$, where the superscript c denotes the complement, and $m=\sum_{k} m_{k}$. Thus $T$ is the set of trapped points, $T^{\prime}$ is $T$ with the pre-image points added, $S$ is the set of stochastic points, $S^{\prime}$ is the $S$ with the pre-image points removed, and $m$ is the total number of trapped points. It is easy to generalize $(\mathrm{C} 1)$ to incorporate the additional trapped segments.

If $\mathbf{F}(t)$ is a column vector giving the distribution function at time $t$, then

$$
\mathbf{F}(t)=\mathbf{P}^{t} \mathbf{F}(0) \text {. }
$$

As $t \rightarrow \infty, \mathbf{P}^{t}$ approaches a matrix $\mathbf{C}$ each of whose entries is the constant $1 / N$. It is convenient to define $\mathbf{Q}$ such that $\mathbf{Q}=\mathbf{P}-\mathbf{C}$. It is easy to show that, for $t>0, \mathbf{Q}^{t}=\mathbf{P}^{t}-\mathbf{C}$ so that $\lim _{t \rightarrow \infty} \mathbf{Q}^{t}=\mathbf{0}$. Since so many of the entries in $\mathbf{Q}^{t}$ are the same, the computational effort involved in doing the matrix multiplication is the same as for multiplying $(m+1) \times$ $(m+1)$ matrices.

The correlation function for a function $h$ on phase space is given by

$$
\mathcal{C}(\tau)=\mathbf{h} \cdot \mathbf{P}^{\tau} \cdot \mathbf{h} / N,
$$

where $\mathbf{h}$ is a vector giving the value of $h$ for each point. This parallels the definition (13) given in section 6 . As in that section, we will assume that $\sum h_{j}=0$. In that case, we have $\mathbf{h} \cdot \mathbf{C} \cdot \mathbf{h}=0$, so that we can replace $\mathbf{P}$ by $\mathbf{Q}$ in this definition.

Let us consider first the case where there is a single trapped segment, $M=1$. Suppose that $h_{j}$ is 1 for $j \in T, 0$ for $j=q_{1}$, and takes on arbitrary values (consistent with the requirement that $\left.\sum h_{j}=0\right)$ for $j \in S^{\prime}$. In the example given in (C1), we might choose $\mathbf{h}=[1,1,1,-1,1,-3,0]$. (We pick $h$ to be zero for the pre-image point, so that there is no bias for this point.) In the notation of section 6 , we have $A_{1}=N, B_{1}=m, f_{t}^{*}=\delta_{t m} / m$, $\mathcal{C}_{\text {is }}(\tau)=(m-\tau) / N$ for $\tau \leq m, \mathcal{D}_{\text {is }}=\frac{1}{2} m^{2} / N$.

We have numerically determined $\mathcal{C}(\tau)$ for this case using $(\mathrm{C} 2)$ with $m=10,20$, and 50 and $N$ varying between $10 \mathrm{~m}$ and $10^{6} \mathrm{~m}$. In particular, we considered $\epsilon(\tau) \equiv(\mathcal{C}(\tau)-$ $\left.\mathcal{C}_{\text {is }}(\tau)\right) / \mathcal{C}_{\text {is }}(0)$. We found that for $\tau>0,|\epsilon(\tau)| \leq m / N=B_{1} / A_{1}$. After an initial transient, $\epsilon(\tau)$ settles down to a decaying periodic function, which oscillates about zero, whose halfperiod is $m$, and which decays as $n^{-\tau / m}$ approximately. Thus for $N \gg m$, the corrections to $\mathcal{C}_{\text {is }}(\tau)$ are very small.

It is, perhaps, a little surprising that this model predicts anti-correlations where $\mathcal{C}(\tau)<$ 0 . This is most pronounced for $m \lesssim \tau \lesssim 2 m$. It is, however, a reflection of a real effect in mappings. Consider an orbit in the stochastic sea of a general mapping. If there is an island present, the trajectory can be stuck close to the island for very long times. However, because of ergodicity, the average time the orbit spends close to the island is equal to the relative areas of the stochastic components $B_{1} / A_{1}$. Thus, to compensate for the stickiness, an orbit far from the island must have some difficulty coming close to the island. This leads to the anti-correlations observed. (These anti-correlations may be rather difficult to measure directly because the presence of trapped segments of so many different lengths will average out the oscillations in $\mathcal{C}$.) A fairly accurate picture of the situation is that the stochastic region close to the island is surrounded by a box with a very small opening in 
it. A particle inside the box has to bounce around a lot before finding the opening and escaping. Similarly, once the particle is outside the box (in the main part of the stochastic sea), it cannot easily find the opening in order to come close to the island once again.

Because $\mathcal{C}_{\text {is }}(\tau)=0$ for $\tau \geq m$, whereas $\epsilon(\tau)$ is finite for all times, we still need to verify that the integrated effect of $\epsilon(\tau)$ (which gives the corrections to $\mathcal{D}_{\text {is }}$ ) is small. We define the diffusion coefficient in the same way as (6). This gives

$$
\mathcal{D}=\mathbf{h} \cdot\left(\frac{1}{2} \mathbf{l}+\sum_{\tau=1}^{\infty} \mathbf{Q}^{\tau}\right) \cdot \mathbf{h} / N=\mathbf{h} \cdot\left(\frac{1}{2} \mathbf{l}+\mathbf{Q} \cdot(\mathbf{I}-\mathbf{Q})^{-1}\right) \cdot \mathbf{h} / N
$$

We are able to write the sum in this way because $\mathbf{Q}^{\tau}$ converges to zero. The the matrix operations were performed for several examples with 1 or 2 trapped segments using MACSYMA [16]. The results show that in general $\mathcal{D}$ may be written as

$$
\mathcal{D}=\frac{1}{2 N}\left(\sum_{j \in S^{\prime}} h_{j}^{2}+\sum_{k} \sum_{i \in T_{k}^{\prime}} \sum_{j \in T_{k}^{\prime}} h_{i} h_{j}\right) .
$$

This result is exact and only requires that $\sum h_{j}=0$. The first term in parentheses is just the contribution from $\mathcal{C}(0)$ for the stochastic points, while the second term gives island contribution. In the one-segment case discussed above, the island contribution clearly reduces to $\frac{1}{2} m^{2} / N$ which exactly equals $\mathcal{D}_{\text {is }}$. Thus the oscillations in $\epsilon(\tau)$ are such that its sum is precisely zero.

For the more general case, where there is an arbitrary number of trapped segments of various lengths, there are two requirements: $h_{j}$ should be a constant $h_{0}$ for $j \in T$ and $\sum_{k} m_{k} h_{q_{k}}$ should be zero. (This last condition says that the pre-image points should be sufficiently evenly spread over phase space.) From section 6 , we have $f_{t}^{*}=\sum_{k} \delta_{t m_{k}} / m$, $\mathcal{D}_{\text {is }}=\frac{1}{2} h_{0}^{2} / N \sum_{k} m_{k}^{2}$, while from (C3) we obtain

$$
\mathcal{D}=\frac{1}{2 N}\left(\sum_{j \in S} h_{j}^{2}+h_{0}^{2} \sum_{k} m_{k}^{2}\right) .
$$

Again, the second term exactly gives $\mathcal{D}_{\text {is }}$. Alternatively, we could declare that the pre-image points are part of the trapped segments. (This conflicts with the picture given in section 6 because it would allow one trapped segment to follow immediately after another with no intervening free segment.) Letting $h_{j}=h_{0}$ for $j \in T^{\prime}$ and defining $m_{k}^{\prime}=m_{k}+1$, (C4) would be modified by replacing $S$ by $S^{\prime}$ and $m_{k}$ by $m_{k}^{\prime}$. 


\section{References}

[1] B. V. Chirikov, Phys. Rept. 52 (1979) 263.

[2] A. B. Rechester and R. B. White, Phys. Rev. Lett. 44 (1980) 1586.

[3] C. F. F. Karney, A. B. Rechester, and R. B. White, Physica 4D (1982) 425.

[4] S. R. Channon and J. L. Lebowitz, Numerical Experiments in Stochasticity and Homoclinic Oscillation, in Nonlinear Dynamics, Annals of the New York Academy of Sciences 357 (New York, 1980) 108; S. R. Channon, Stochasticity and Homoclinic Oscillations in an Area-Preserving Mapping, Doctoral Thesis, Rutgers University (1981).

[5] B. V. Chirikov and D. L. Shepelyansky, Statistics of Poincaré Recurrences and the Structure of the Stochastic Layer of a Nonlinear Resonance (in Russian), 9th International Conference on Nonlinear Oscillations, Kiev, 1981, Institute of Nuclear Physics Preprint INP 81-69, Novosibirsk (1981); [English translation available as Plasma Physics Laboratory Report PPPL-TRANS-133, Princeton University (1983)].

[6] J. D. Meiss, J. R. Cary, C. Grebogi, J. D. Crawford, A. N. Kaufman, and H. D. I. Abarbanel, Physica 6D (1983) 375.

[7] J. M. Greene, R. S. MacKay, F. Vivaldi, and M. J. Feigenbaum, Physica 3D (1981) 468.

[8] R. S. MacKay, Physics Lett. 87A (1982) 321 and Renormalization in Area Preserving Maps, Doctoral Thesis, Princeton University (1982).

[9] M. Hénon, Quarterly of App. Math. 27 (1969) 291.

[10] A. B. Zisook, Phys. Rev. A25 (1982) 2289.

[11] J. M. Greene, J. Math. Phys. 20 (1979) 1183.

[12] J. M. Greene, private communication (1982).

[13] B. V. Chirikov, Chaotic Dynamics in Hamiltonian Systems with Divided Phase Space, 7th Sitges Conference on "Dynamical Systems and Chaos", Barcelona, 1982, Institute of Nuclear Physics Preprint INP 82-132, Novosibirsk (1982).

[14] F. Rannou, Astron. and Astrophys. 31 (1974) 289 and Etude Numérique de Transformations Planes Discrètes (in French), Doctoral Thesis, University of Nice (1972).

[15] D. E. Knuth, The Art of Computer Programming, 2nd Edition, Vol. 2 (Addison Wesley, 1981).

[16] The Mathlab Group, MACSYMA Reference Manual, Version 9, Laboratory for Computer Science, Massachusetts Institute of Technology (1977). 


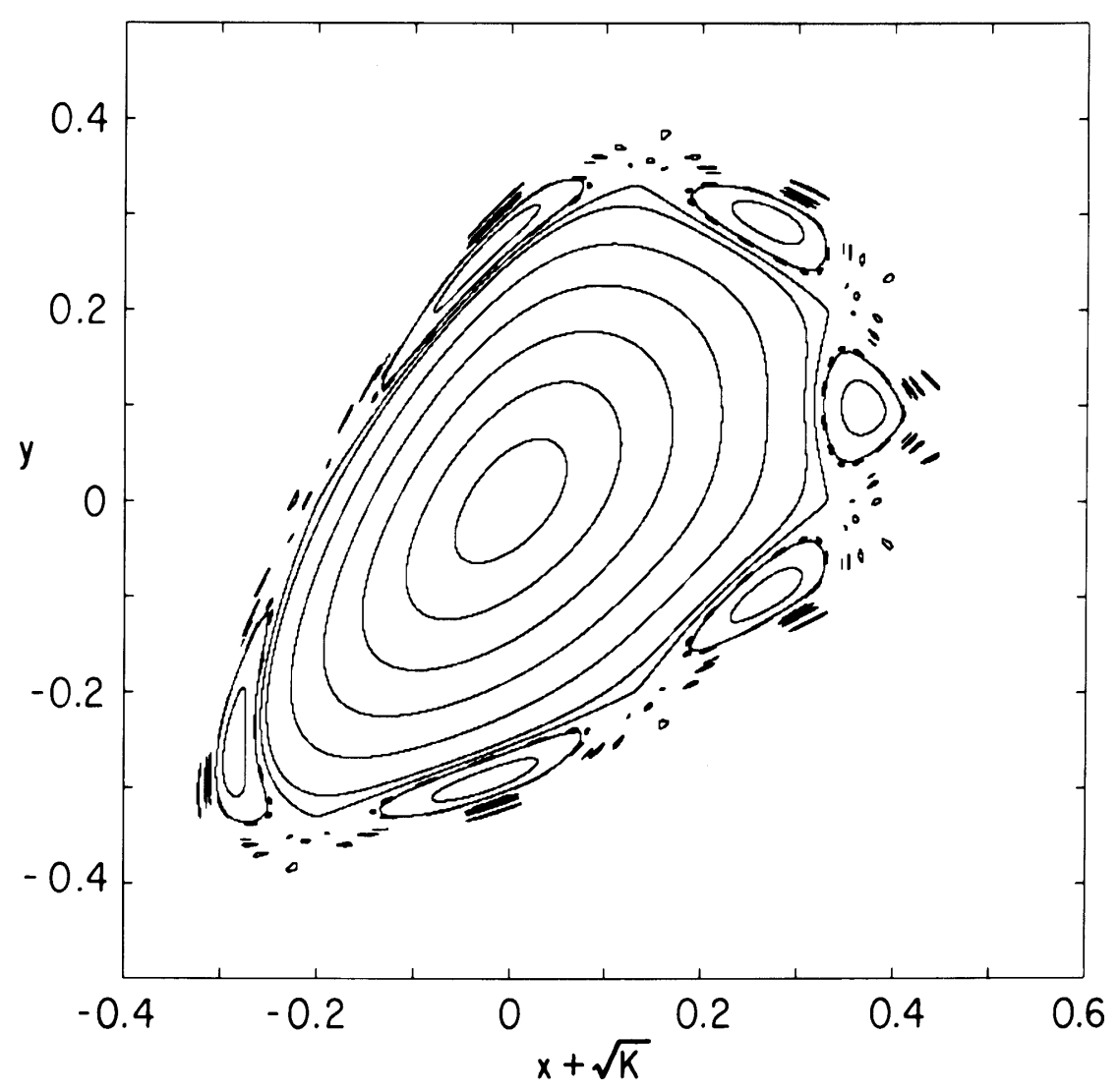

Fig. 1. Some islands of the quadratic map $Q(2)$ for $K=0.1$. 

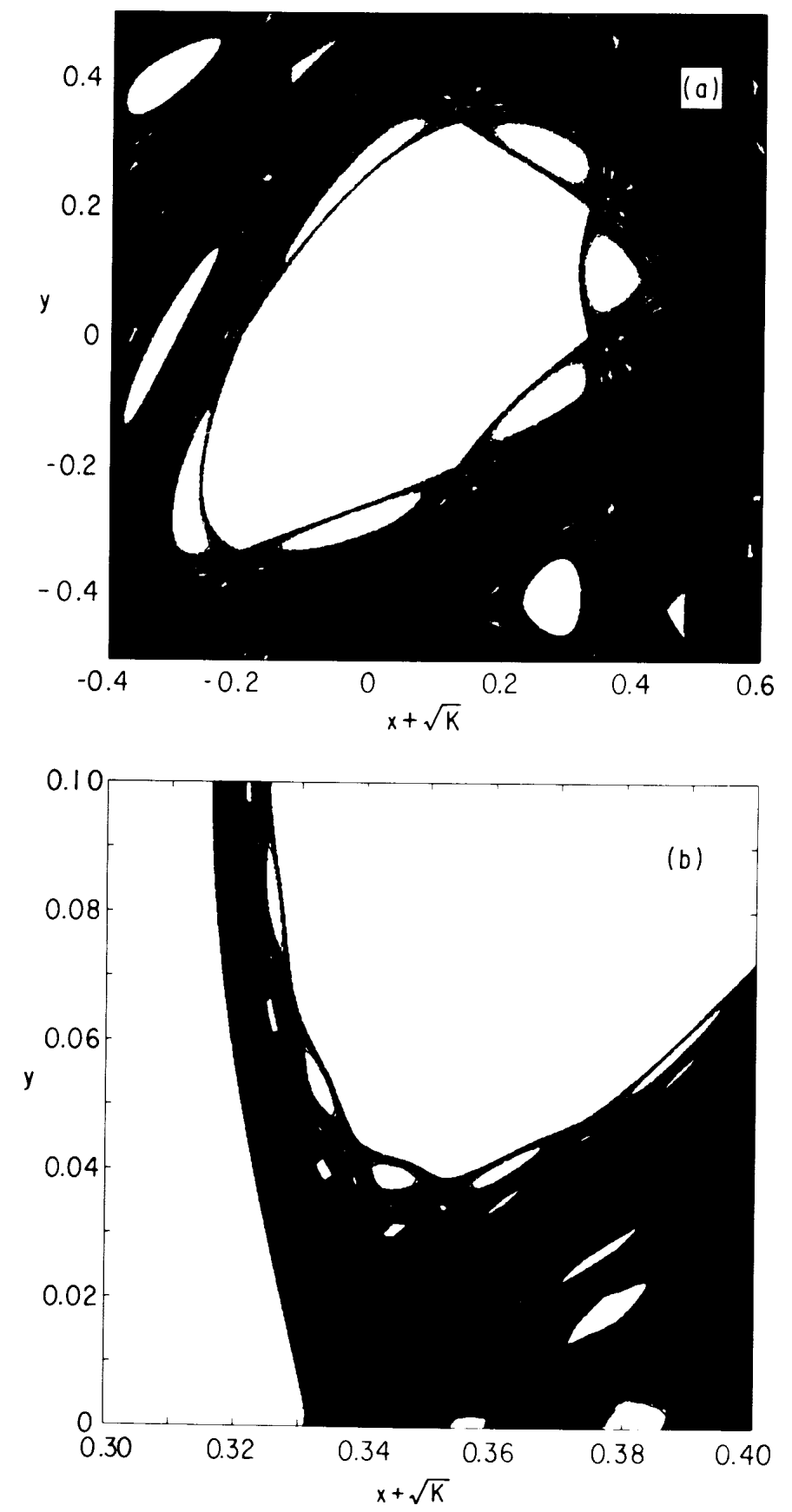

Fig. 2. (a) Stochastic trajectories for periodic quadratic mapping $Q^{*}$ for $K=0.1$. (b) An enlargement of a portion of (a). Here $x_{\min }+\sqrt{K}=-0.4, x_{\max }+\sqrt{K}=0.6, X_{r}=0.02$. (a) was produced by plotting every 1000th point of 64 orbits each of length $10^{7}$ and (b) by plotting every 100th point of 64 orbits of length $2 \times 10^{8}$. 

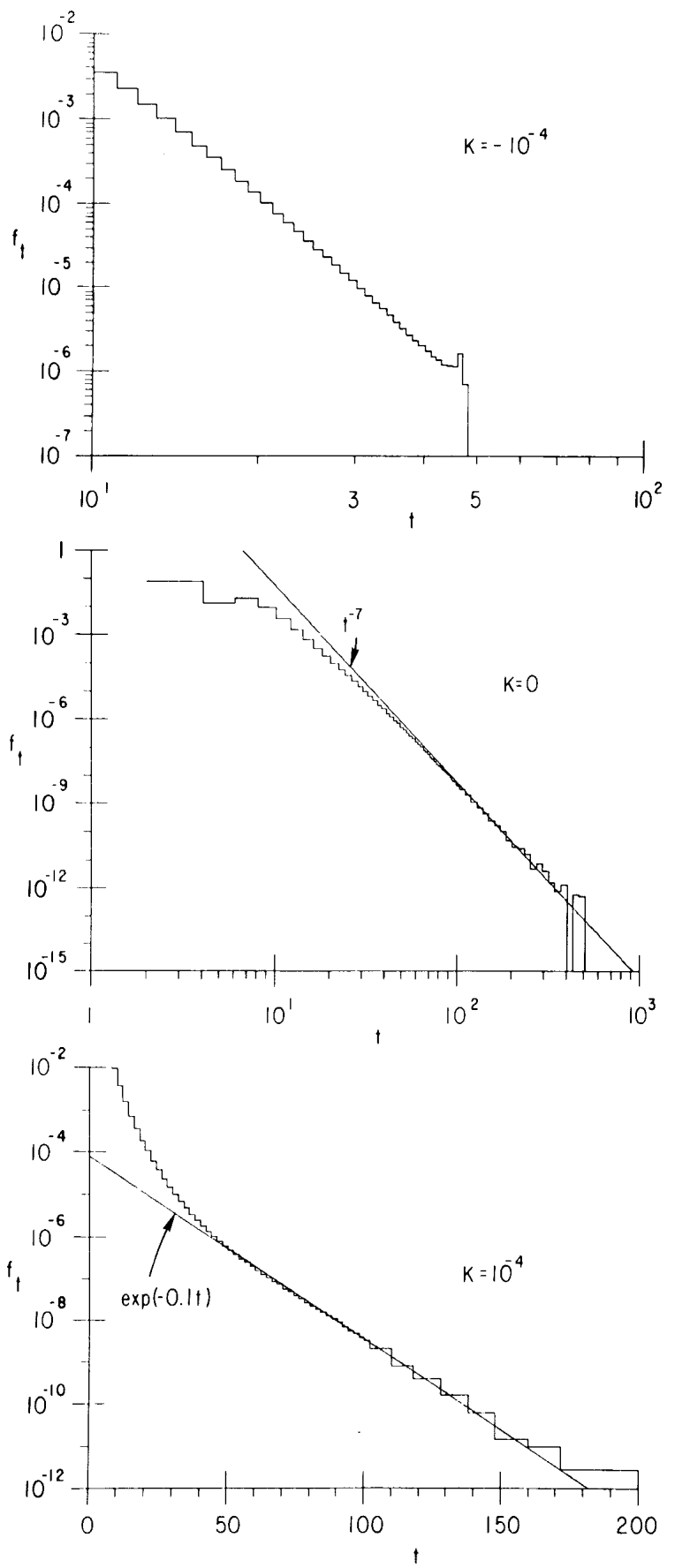

Fig. 3. The trapping statistics $f_{t}$ for (a) $K=-10^{-4}$, (b) $K=0$, and (c) $K=10^{-4}$. In each case $x_{\min }+\sqrt{\max (K, 0)}=-0.5, x_{\max }+\sqrt{\max (K, 0)}=0.5, X_{r}=0.02$. For (a) $M=128, q=1, q T=5 \times 10^{7}$; for (b) and (c) $M=512, q=2, q T=10^{8}$. In (b) and (c) the straight lines give the $t^{-7}$ and $\exp (-0.1 t)$ behaviors predicted in section 4 . 

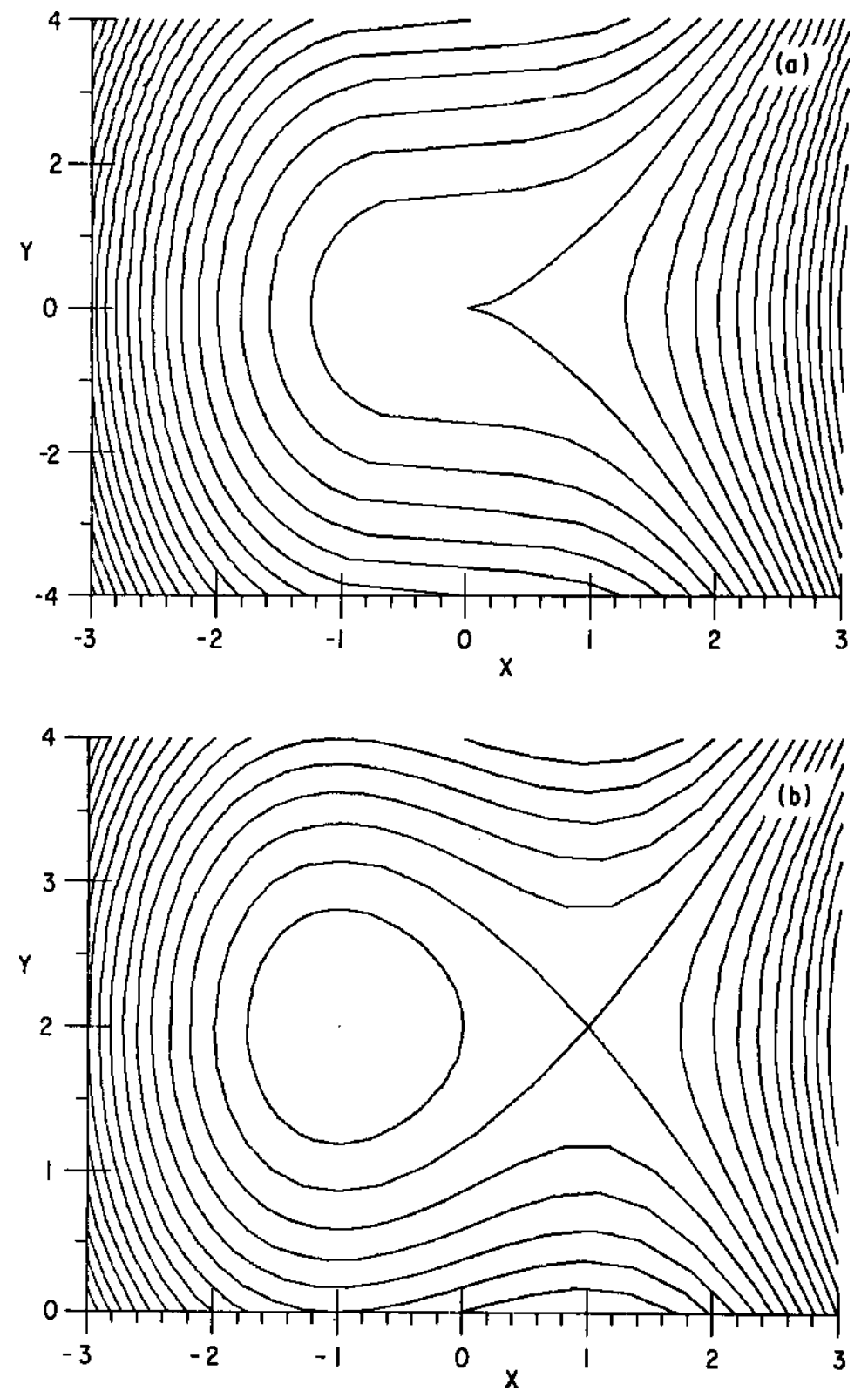

Fig. 4. Trajectories, for the Hamiltonian with (a) $K=0$ (7) and with (b) $K$ small and positive (11). In both figures $H$ takes on equally spaced values with an increment of $\frac{4}{3}$. 

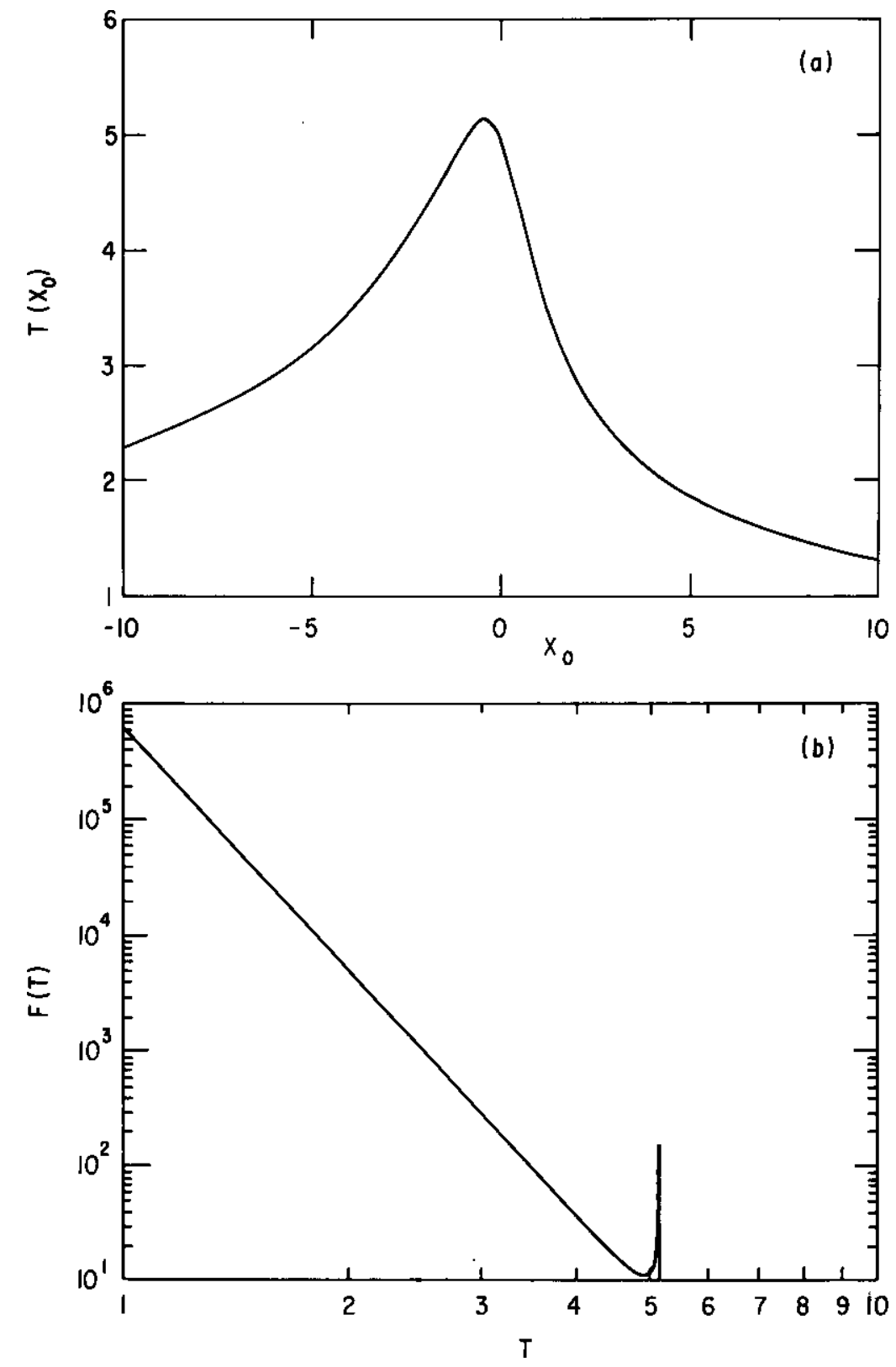

Fig. 5. (a) The trapping time $T\left(X_{0}\right)$ for $K<0(9)$. (b) $F(T)$ as a function of $T$ (10). 

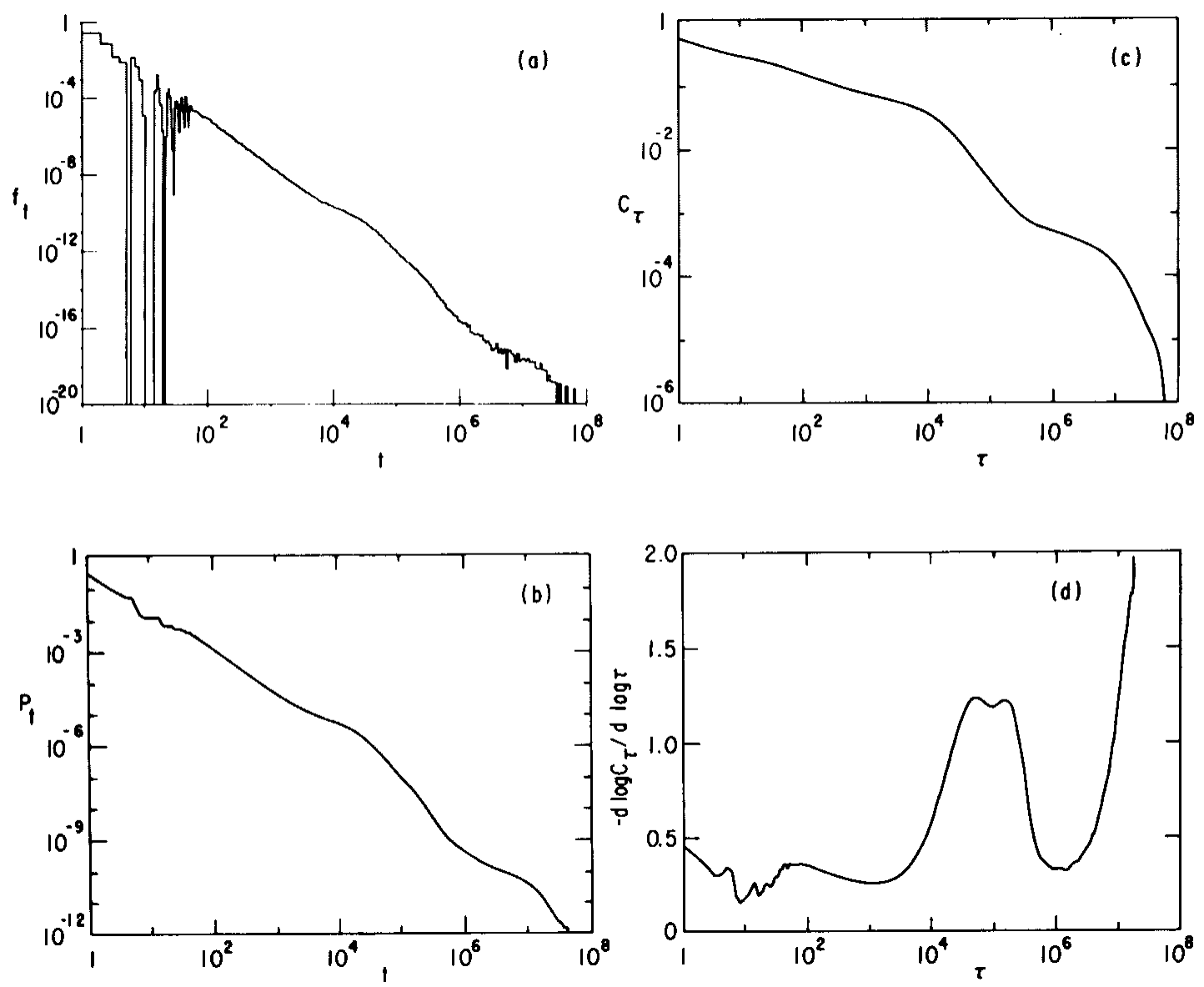

Fig. 6. (a) The trapping statistics $f_{t}$ for $K=0.1$. (b), (c), and (d) show $P_{t}, C_{\tau}$, and $d \log C_{\tau} / d \log \tau$ for the same case. Here $x_{\min }+\sqrt{K}=-0.4, x_{\max }+\sqrt{K}=0.6, X_{r}=0.02$. The data for $f_{t}$ was obtained in 3 pieces; for $1 \leq t<10^{3}, M=128, q=1, q T=5 \times 10^{7}$; for $10^{3} \leq t<10^{5}, M=256, q=10, q T=5 \times 10^{8}$; for $10^{5} \leq t, M=1600, q=1000$, $q T=2 \times 10^{9}$. 

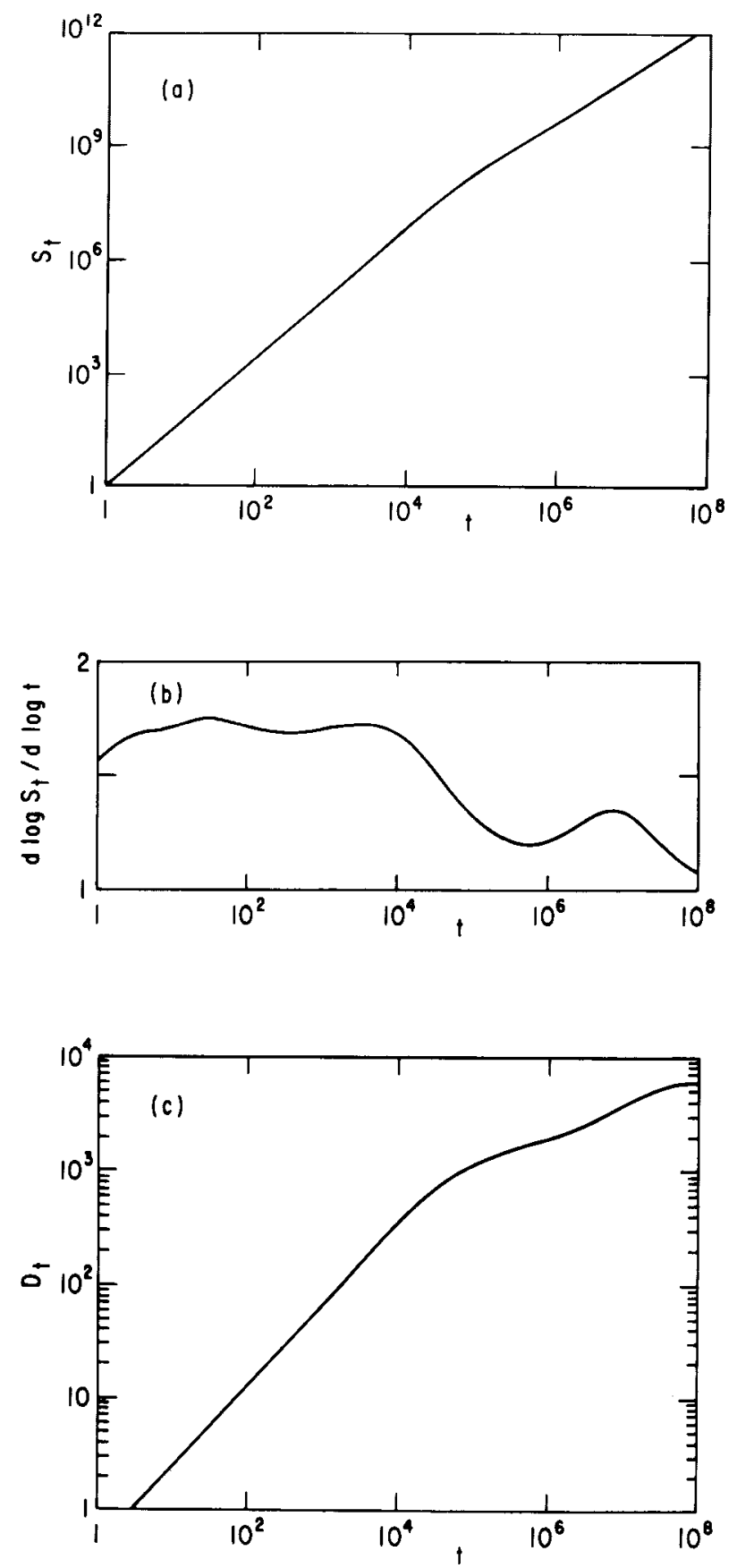

Fig. 7. (a) The variance $S_{t}$ for the case given in fig. 6. (b) and (c) show $d \log S_{t} / d \log t$ and $D_{t}=\frac{1}{2} S_{t} / t$. 


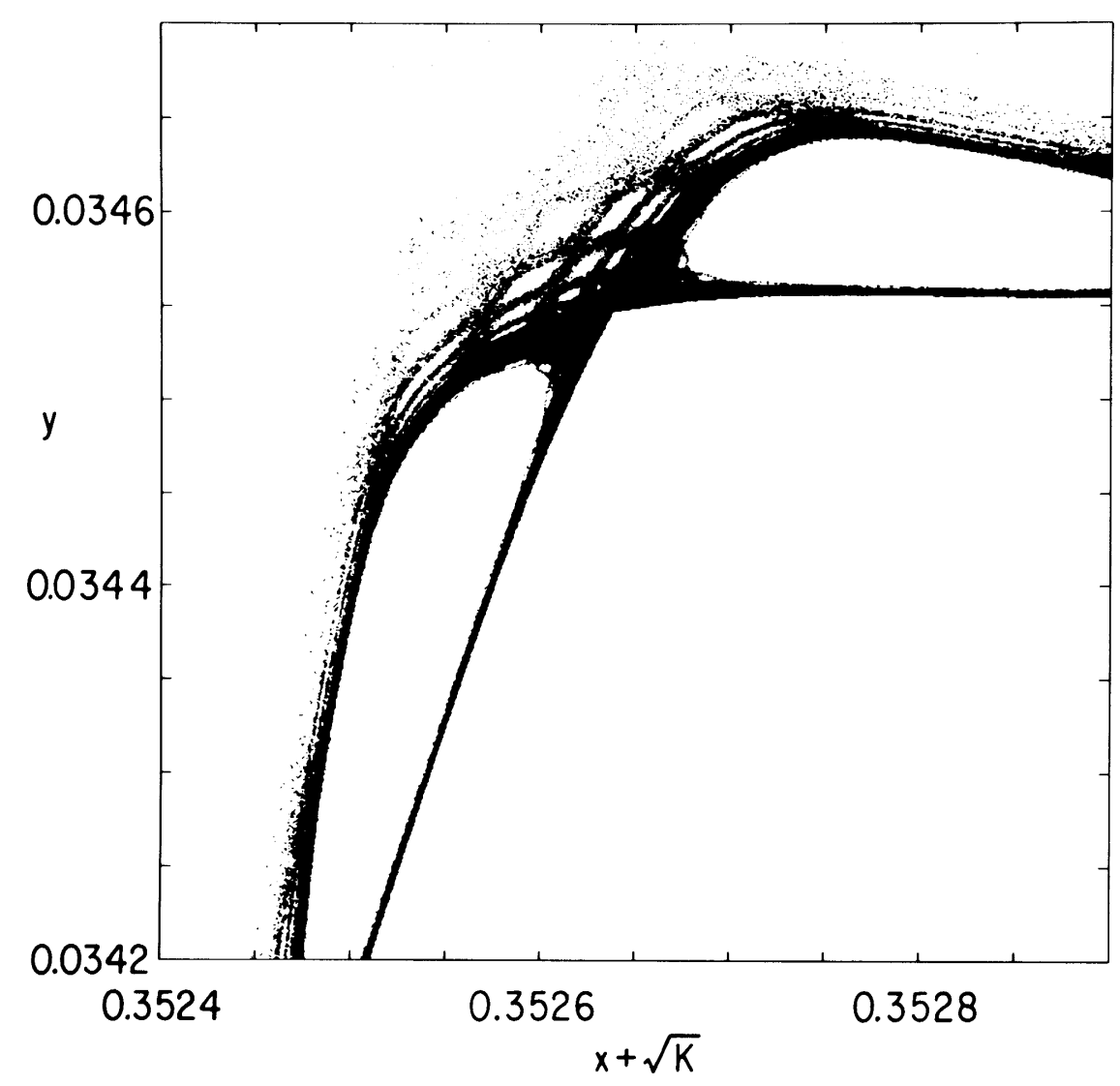

Fig. 8. An enlargement of a long orbit segment in fig. 6(a). The bottom left part of the picture is occupied by a member of a chain of 138th-order islands. 